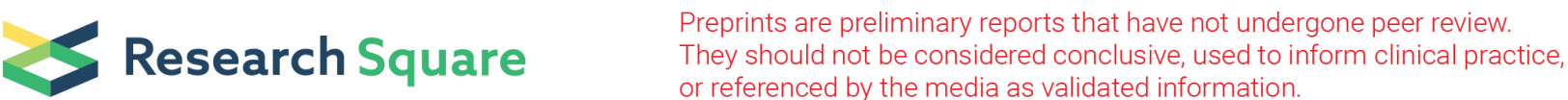

\section{EZH2 Inhibition Sensitizes BRCA1-Deficient Breast Cancer to Synthetic Lethal Therapy with ATM Inhibitors}

Leonie Ratz ( $\square$ leonie.ratz@uk-koeln.de )

University Hospital Cologne: Uniklinik Koln https://orcid.org/0000-0002-4783-987X

Chiara Brambillasca

Netherlands Cancer Institute: Antoni van Leeuwenhoek Nederlands Kanker Instituut

Leandra Bartke

University Hospital Cologne: Uniklinik Koln

\section{Marieke van de Ven}

Netherlands Cancer Institute: Antoni van Leeuwenhoek Nederlands Kanker Instituut

\section{Natalie Proost}

Netherlands Cancer Institute: Antoni van Leeuwenhoek Nederlands Kanker Instituut

\section{Bjørn Siteur}

Netherlands Cancer Institute: Antoni van Leeuwenhoek Nederlands Kanker Instituut

\section{Renske de Korte-Grimmerink}

Netherlands Cancer Institute: Antoni van Leeuwenhoek Nederlands Kanker Instituut

\section{Peter Bouwman}

Leiden University: Universiteit Leiden

\section{Emilia M. Pulver}

Netherlands Cancer Institute: Antoni van Leeuwenhoek Nederlands Kanker Instituut

\section{Roebi de Bruijn}

Netherlands Cancer Institute: Antoni van Leeuwenhoek Nederlands Kanker Instituut

Jörg Isensee

University Hospital Cologne: Uniklinik Koln

\section{Tim Hucho}

University Hospital Cologne: Uniklinik Koln

\section{Gaurav Pandey}

Netherlands Cancer Institute: Antoni van Leeuwenhoek Nederlands Kanker Instituut

\section{Maarten van Lohuizen}

Netherlands Cancer Institute: Antoni van Leeuwenhoek Nederlands Kanker Instituut

\section{Peter Mallmann}

University Hospital Cologne: Uniklinik Koln

\section{Hans Christian Reinhardt}


University Hospital Essen: Universitatsklinikum Essen

Jos Jonkers

Netherlands Cancer Institute: Antoni van Leeuwenhoek Nederlands Kanker Instituut

Julian Puppe

University Hospital Cologne: Uniklinik Koln

\section{Research article}

Keywords: BRCA1 mutation, synthetic lethality, EZH2, breast cancer

Posted Date: June 15th, 2021

DOI: https://doi.org/10.21203/rs.3.rs-580939/v1

License: (9) This work is licensed under a Creative Commons Attribution 4.0 International License. Read Full License 


\section{Abstract}

Background: The majority of BRCA1-mutant breast cancers are characterized by a triple-negative phenotype (TNBC) and a basal-like molecular subtype, associated with aggressive clinical behavior. Current treatment options are limited, highlighting the need for the development of novel targeted therapies for this tumor subtype.

Methods: Our group previously showed that EZH2 is functionally relevant in BRCA1-deficient breast tumors and blocking EZH2 enzymatic activity could be a potent treatment strategy. To validate the role of $\mathrm{EZH} 2$ as a therapeutic target and to identify new synergistic drug combinations, we performed a highthroughput drug combination screen in various cell lines derived from BRCA1-proficient and deficient mouse mammary tumors.

Results: We identified the combined inhibition of EZH2 and the proximal DNA damage response kinase ATM as a novel synthetic lethality-based therapy for the treatment of BRCA1-deficient breast tumors. We show that the combined treatment with the EZH2 inhibitor GSK126 and the ATM inhibitor AZD1390 led to reduced colony formation, increased genotoxic stress, and apoptosis-mediated cell death in BRCA1deficient mammary tumor cells in vitro. These findings were corroborated by in vivo experiments showing that simultaneous inhibition of EZH2 and ATM significantly increased anti-tumor activity in mice bearing BRCA1-deficient mammary tumors.

Conclusion: Taken together, we identified a synthetic lethal interaction between EZH2 and ATM and propose this synergistic interaction as a novel molecular combination for the treatment of BRCA1-mutant breast cancer.

\section{Introduction}

Triple-negative breast cancer (TNBC) is the most aggressive phenotype of breast cancer with limited treatment options and unfavorable prognosis. TNBCs encompass heterogeneous molecular subtypes, and $20-30 \%$ are associated with disabling germline BRCA1/2 mutations (gBRCA) and a basal-like molecular subtype (1). Moreover, $25 \%$ of sporadic TNBCs are characterized by (epi)genetic defects in homologous recombination (HR), that induce pathological and molecular changes resembling $B R C A 1 / 2$ mutation-associated cancers (BRCAness) (2-4). TNBCs frequently show an inadequate response to (neo)adjuvant chemotherapy with poor survival benefit. Dose-dense neoadjuvant chemotherapy is highly recommended and the addition of carboplatin to the standard anthracycline- and taxane-based regimen of patients with TNBC increases the rate of pathological complete remission (pCR), a crucial surrogate of overall survival (5). Highest $\mathrm{pCR}$ rates are achieved in patients with germline BRCA7-mutant breast cancer due to increased genomic instability, however, no overall survival benefit was observed (6-9). Thus, a major effort in clinical breast cancer research is to define a therapeutic algorithm with novel treatments for TNBC. 
At the cellular level, the BRCA1 gene product is important for DNA double-strand break (DSB) repair through the HR pathway. BRCA1-deficient cells display inappropriate DSB repair due to HR-deficiency. Consequently, those cells depend on the PARP1-mediated base excision repair pathway and nonhomologous end joining (NHEJ) to resolve DNA damage, however, the error-prone character of NHEJ promotes chromosomal rearrangements and genomic instability. Checkpoint gene inactivation (by p53 inactivation, for example) disables cells to induce cell cycle arrest and further accelerates breast cancer development $(10,11)$. Identifying the molecular pathogenesis of BRCA1-mutant TNBCs has directed the implementation of PARP inhibitors in locally advanced and metastatic breast cancer (12). However, durable clinical response to PARP inhibition is counteracted by PARP inhibitor resistance (13). The identification of novel molecular targets for a tailored treatment approach is thus of high importance to increase therapeutic options for patients with BRCA1-mutant breast cancer.

Recent work from our group and others has highlighted histone methyltransferase EZH2 (Enhancer of zeste homolog 2) as a promising target in BRCA1-deficient breast cancer (14-16). EZH2 is the catalytic subunit of the Polycomb repressive complex 2 (PRC2) responsible for histone $\mathrm{H} 3$ lysine 27 trimethylation (H3K27me3) and transcriptional silencing of target genes. EZH2 is involved in various biological functions, including cell cycle control, proliferation, and differentiation in various cancer types (17, 18). BRCA1 has been reported to be a negative regulator of EZH2 (15). Loss of BRCA1 function was shown to increase EZH2 activity by promoting genome-wide EZH2 recruitment to chromatin. This caused an increase in H3K27me3 levels at known PRC2 target loci that have been associated with reduced cellular differentiation and an aggressive breast cancer phenotype (15). EZH2 is overexpressed in tumors with loss of BRCA1 function or a BRCA1-like DNA copy number profile $(14,19,20)$. Patients with BRCA1mutant breast cancer and high EZH2 expression showed an improved therapeutic response and less frequent disease recurrence after treatment with intensified platinum-based chemotherapy, compared to standard chemotherapy (14). Combined treatment with the EZH2 inhibitor GSK126 and cisplatin led to increased cytotoxicity and reduced colony formation in BRCA1-deficient mouse mammary tumor cells (14). Effective in vivo anti-tumor activity of combined GSK126 and cisplatin treatment also enhanced overall survival of mice bearing BRCA1-deficient mouse mammary tumors, suggesting that EZH2 inhibition enhances the sensitivity to platinum drugs in EZH2-overexpressing breast tumors (14).

Epigenetic synthetic lethal approaches have gained interest in cancer therapies, due to various aberrant epigenetic regulatory mechanisms leading to actionable dependencies (21). Several synthetic lethal partners of EZH2 or mutations conferring EZH2 dependence have been identified in hematological malignancies and solid cancers, such as breast, ovarian, and non-small cell lung cancer (22-26; reviewed by 21). Since BRCA1-mutant breast cancers harbor a defect in their DNA repair machinery leading to a dependency on alternative repair pathways, synthetic lethal therapies show high potential in this genetic context as highlighted by the clinical use of PARP inhibitors to treat BRCA1/2-mutant breast and ovarian cancer $(12,27,28)$. We therefore aimed to identify synthetic lethal partners of EZH2 in BRCA1-deficient breast cancer. 


\section{Results}

\section{High-throughput drug screening identifies synergistic cytotoxicity of EZH2 and ATM inhibition in a BRCA1-deficient context}

Previously, we reported that $\mathrm{EZH} 2$ is highly expressed in tumors associated with a BRCAness profile. Here, the association of $E Z H 2$ mRNA expression with BRCA1-mutant breast cancer was further evaluated using the TCGA BRCA whole exome sequencing data set $(n=526)$ confirming the increased expression of $E Z H 2$ in BRCA1-mutant $(\mathrm{n}=18)$, compared to BRCA1-wild type breast cancer $(\mathrm{n}=508)(\mathrm{p}=0.0003$; Fig. $1 \mathrm{~A}$, left panel). Significantly increased Ezh2 expression in female BRCA1-

deficient $\mathrm{K} 14 \mathrm{Cre} ; \mathrm{Brca} 1^{\mathrm{F} / \mathrm{F}} ; \operatorname{Trp}^{\mathrm{F}} 3^{\mathrm{F} / \mathrm{F}}$ (KB1P) mouse mammary tumors compared to BRCA1proficient $\mathrm{K} 14 \mathrm{cre}, \operatorname{Trp} 53^{\mathrm{F} / \mathrm{F}}(\mathrm{KP})$ mammary tumors was further corroborated by RNA sequencing of tumors ( $p=0.0156$; Fig. $1 \mathrm{~A}$, right panel).

To study the role of EZH2 in BRCA1-mutant breast cancer, we made use of previously described murine tumor cell lines derived from KB1P (clones KB1P-G3 and KB1P-B11) or KP (clones KP-3.33 and KP6.3) mammary tumors (29). First, inhibition of the EZH2 methyltransferase activity by GSK126 was assessed, showing reduced H3K27me3 level by Western blotting (Fig. 1B). We then determined the IC $\mathrm{C}_{50}$ value of GSK126 for all cell clones, showing that 3- to 11-fold lower concentrations of GSK126 were required for a $50 \%$ reduction in viability in BRCA1-deficient compared to BRCA1-proficient cells, indicating that BRCA1-deficient mammary tumor cells are more sensitive to EZH2 inhibition (Fig. 1C; KB1P-G3: $\left.\mathrm{IC}_{50}=13.4 \mu \mathrm{M} ; \mathrm{KB} 1 \mathrm{P}-\mathrm{B} 11: \mathrm{IC}_{50}=17.6 \mu \mathrm{M} ; \mathrm{KP}-3.33: \mathrm{IC}_{50}=44.9 \mu \mathrm{M} ; \mathrm{KP}-6.3: \mathrm{IC}_{50}=148.9 \mu \mathrm{M}\right)$.

In order to identify synthetic lethal partners of EZH2 that are specifically effective in BRCA1-deficient breast cancer, we used BRCA1-deficient and -proficient mammary tumor cell lines to perform a highthroughput drug screen in combination with GSK126. We analyzed a panel of 27 distinct compounds targeting main oncogenic signaling pathways, such as DNA repair and cell cycle checkpoint signaling, and included small-molecule inhibitors that are in preclinical testing for cancer treatments. Single agent profiles for $\mathrm{IC}_{50}$ determination were assessed in serial dilutions for all compounds in BRCA1-deficient (KB1P-G3 and KB1P-B11) and BRCA1-proficient (KP-3.33 and KP-6.3) cells (Supplementary Fig. S1 and Supplementary Table S1). Each compound was then profiled for synergistic interaction with GSK126, using $6 \times 6$ concentration combinations (vehicle control, 5 single concentrations per compound, 25 combinations). For analysis of potential synergy, the Bliss independence model was used with a score of $>15$ indicative of a synergistic compound interaction (Supplementary Table S1). Synergy scores were further analyzed based on the difference between BRCA1-deficient and BRCA1-proficient cell lines. Synergistic compound screening highlighted the two Ataxia telangiectasia mutated (ATM) inhibitors AZD1390 (30) and KU60019 (31) as the best hits with the highest difference in synergy scores between BRCA1-deficient and -proficient cells (Fig. 1D). Synergistic effects were visualized using the webapplication tool SynergyFinder (32). Heatmaps for GSK126/AZD1390 and GSK126/KU60019 combination treatments are depicted in Fig. 1E. Visualized synergy heatmaps and synergy scores for the remaining compounds in combination with GSK126 are shown in Supplementary Fig. S2 and Table S1, 
respectively. Since the highest synergy score was observed for AZD1390 (Bliss synergy score: 26.6 in KB1P-G3, 22.9 in KB1P-B11), this ATM-kinase specific inhibitor was prioritized for further analysis in combination with GSK126.

ATM together with ATR (ataxia telangiectasia and Rad3 related) are DNA damage sensor kinases that, upon induction of DNA damage, phosphorylate checkpoint kinase 1 and 2 (CHK1/2), respectively (33). This results in G1-S and G2-M cell cycle checkpoint activation and initiation of DNA repair. ATM is a central molecule in the signaling cascade of DSBs. In the absence of an intact DNA repair mechanism, collateral ATM-deficiency leads to increased genomic instability (34).

The ATM inhibitor AZD1390 was able to inhibit downstream ATM signaling as shown by reduced Kap1 (S824) phosphorylation (Fig. 2A-B). AZD1390 showed 4- to 6-fold lower IC 50 values for BRCA1-deficient cells than BRCA1-proficient cells, indicating that BRCA1-deficient cells are more sensitive to ATM inhibition (Fig. 2C; KB1P-G3: IC ${ }_{50}=6.70 \mu \mathrm{M}$; KB1P-B11: IC ${ }_{50}=6.8 \mu \mathrm{M} ; \mathrm{KP}-3.33$ : IC ${ }_{50}=26.9 \mu \mathrm{M} ; \mathrm{KP}-6.3$ : $\left.\mathrm{IC}_{50}=42.90 \mu \mathrm{M}\right)$.

\section{In vitro optimization of the synergistic effect from combined EZH2/ATM inhibition}

In addition to genetic context, the choice of compound concentration is crucial to achieve maximal drug synergy and to avoid over- or under-treatment resulting in unspecific toxicity or response failure (35). Therefore, a concentration optimization experiment was performed to determine the compound concentration with the highest synergy between GSK126 and AZD1390. BRCA1-deficient and -proficient mouse mammary tumor cells were treated with increasing concentrations of one compound in the absence or presence of a fixed concentration of the other compound and vice versa (Fig. 2D). Single agent GSK126 treatment at intermediate concentrations induced moderate cytotoxicity in BRCA1deficient cells, but strong cytotoxicity with the highest concentration of $10 \mu \mathrm{M}$ (Fig. 2D, upper panels; Supplementary Fig. S3A). The cytotoxic effect could be potentiated by adding a fixed concentration of AZD1390 at $2 \mu \mathrm{M}$, while this concentration had no cytotoxic effect as a single treatment (Fig. 2D, lower panels). Treatment with increasing concentrations of single agent AZD1390 slightly decreased BRCA1deficient cell viability, while no cytotoxic effect was observed in BRCA1-proficient cells (Fig. 2D, lower panels). When introducing GSK126 at a fixed concentration of $7.5 \mu \mathrm{M}$, BRCA1-deficient cells were sensitized to the cytotoxic effect of AZD1390 $(2 \mu \mathrm{M})$, while BRCA1-proficient cells remained unaffected by this combination. As was evident from the $\mathrm{IC}_{50}$ determination, high concentrations of single GSK126 or AZD1390 treatment also induced toxicity in BRCA1-proficient cells (Fig. 1C and Fig. 2C), however, this cytotoxicity was not attributed to a synergistic effect but rather to single agent-induced toxicity. Additional data on the concentration optimization analysis are depicted in Supplementary Fig. S3. This experiment revealed maximal synergistic interaction between $7.5 \mu \mathrm{M}$ GSK126 and $2 \mu \mathrm{M}$ AZD1390 in BRCA1-deficient cells, which is below the half-maximal inhibitory concentration of both compounds. These concentrations were thus used for subsequent validation experiments. First, the inhibitory effect on cell viability was validated in an independent cell viability measurement using CellTiter-Glo (Fig. 2E). GSK126 and AZD1390 single agent treatment induced $17 \%$ and $7 \%$ reduction of viability in BRCA1- 
deficient cells, respectively. Remarkably, the combination of both drugs displayed $93 \%$ cytotoxicity in BRCA1-deficient cells (Fig. 2E), while no significant toxicity was observed in BRCA1-proficient cells, confirming that the reduction in cell viability was triggered by context-specific synergistic activity of GSK126 and AZD1390 in BRCA1-deficient cells.

To investigate the cytotoxic effect of combined EZH2 and ATM inhibition in human BRCA1-mutant breast cancer, we treated the human TNBC cell lines SUM149 (BRCA1-mutant) and CAL120 (BRCA1-wild type) with GSK126 and AZD1390 (Fig. 2F). SUM149 cells were specifically sensitive to combined GSK126/AZD1390 inhibition leading to a significantly reduced cell viability by $77-79 \%$, compared to vehicle control $(p=0.02)$ or single agent treatment (GSK126: $p=0.02$; AZD1390: $p=0.03)$, while no effect was observed in CAL120 cells (Fig. 2F), indicating that the cytotoxic effect by EZH2/ATM inhibition is specific to BRCA1 mutation status in human breast cancer cells.

Combined EZH2/ATM inhibitor treatment was subsequently validated using different functional assays. Assessment of clonogenic survival revealed a substantial decrease in the number of colonies by combined GSK126/AZD1390 treatment in BRCA1-deficent mouse mammary tumor cells, compared to vehicle control, as well as to single GSK126 or AZD1390 treatment (Fig. 3A). Quantification of colony formation showed that combined GSK126/AZD1390 treatment caused a 79-81\% decrease in colonyforming units in BRCA1-deficient cells compared to vehicle control (KB1P-G3: $p<0.0001$, KB1P-B11: $p=0.002)$, a 73-86\% decrease compared to single GSK126 treatment (KB1P-G3: $p=0.0001$, KB1P-B11: $p=0.01$ ), and a 50-57\% decrease compared to single AZD1390 treatment (KB1P-G3: $p=0.0001$, KB1P-B11: $p=0.001$ ), but not in BRCA1-proficient cells, indicating a robust synergistic effect in BRCA1-deficient cells (Fig. 3B). Continuous live-cell imaging using IncuCyte analysis revealed that growth inhibition in BRCA1deficient cells through combined GSK126/AZD1390 treatment occurred between 30-40 hours after induction of treatment, resulting in 68-79\% growth reduction after 120 hours in BRCA1-deficient cells, compared to vehicle control (KB1P-G3: $p<0.0001$, KB1P-B11: $p<0.0001$ ) (Fig. 3C), while growth was not affected in BRCA1-proficient cells. Single agent treatment showed a mild inhibitory effect on growth in BRCA1-deficient cells, which was not statistically significant compared to vehicle control.

\section{Cytotoxicity by combined EZH2/ATM inhibition is mediated by apoptosis}

To investigate whether combined EZH2/ATM inhibition induces apoptosis in BRCA1-deficient mouse mammary tumor cells, we measured the percentage of Annexin V/propidium iodide double-positive cells by flow-cytometry (Fig. 3D). Indeed, the percentage of apoptotic cells drastically increased by combined GSK126/AZD1390 inhibition compared to vehicle control or single agent treatment in BRCA1-deficient cells (KB1P-G3: $41.8 \%$ by GSK126/AZD1390 compared to $9.1 \%$ by vehicle $(p<0.0001), 15.1 \%$ by GSK126 $(\mathrm{p}<0.0001)$ and $12.3 \%$ by AZD1390 ( $\mathrm{p}<0.0001)$; KB1P-B11: 41.7\% by GSK126/AZD1390 compared to $10.1 \%$ by vehicle $(p<0.0001), 13.3 \%$ by GSK126 $(p<0.0001)$ and $10.8 \%$ by AZD1390 $(p<0.0001))$. In contrast, the percentage of apoptotic cells in BRCA1-proficient cells did not change significantly upon combined EZH2/ATM inhibition compared to single agent treatment or vehicle control (KP-3.33: 7.2\% by GSK126/AZD1390 compared to $5.3 \%$ by vehicle $(p=0.17), 6.3 \%$ by GSK126 $(p=0.78)$ and $6.7 \%$ by 
AZD1390 ( $\mathrm{p}=0.95) ; \mathrm{KP}-6.3: 8.5 \%$ by GSK126/AZD1390 compared to $4.2 \%$ by vehicle $(\mathrm{p}=0.0004), 4.2 \%$ by GSK126 ( $p=0.0004$, showing a statistically significant, however, biologically no significant difference) and $6.6 \%$ by AZD1390 ( $p=0.14$ ) (Fig. 3D). Together, these results indicate that the synergistic cytotoxicity of combined EZH2/ATM inhibition in BRCA1-deficient mouse mammary tumor cells is mediated by apoptosis.

\section{Cytotoxic effect of combined EZH2/ATM inhibition is stable after compound exchange}

To confirm that the observed treatment effect is due to combined EZH2/ATM inhibition and not caused by any potential off-target effect, BRCA1-deficient and -proficient mouse mammary tumor cells were treated with the structurally distinct EZH2 and ATM inhibitors ZLD1039 (36) and KU60019 (31), respectively (Fig. 4A-B). Combined EZH2/ATM inhibition by GSK126/KU60019 or ZLD1039/AZD1390 treatment reduced the viability of BRCA1-deficient cells compared to vehicle control by $80-90 \%$ (KB1P-G3: $p<0.0001$, KB1P-B11: $p=0.017$ ), and by 42-59\% (KB1P-G3: $p=0.0005, K B 1 P-B 11: p<0.0001)$, respectively, whereas growth of BRCA1-proficient cells was not significantly affected by either combination (Fig. 4A-B). These data indicate that the cytotoxic effects of the drug combinations are resultant of the combined EZH2/ATM inhibition and not due to unspecific compound effects.

\section{Genetic ablation of EZH2 mimics pharmacological H3K27me3 inhibition}

To further establish the role of EZH2 inhibition in sensitizing BRCA1-deficient cells to ATM inhibition and to exclude off-target drug activity, we made use of a doxycycline (Dox)-inducible shRNA-mediated knockdown system (37) allowing stable and inducible Ezh2 knockdown in BRCA1-deficient (KB1P-G3shEzh2) and BRCA1-proficient (KP-3.33-shEzh2) cells. Following seven days of Dox-induced Ezh2 knockdown, a strong decrease in EZH2 protein expression and global H3K27 trimethylation was observed in shEzh2-expressing cells but not in cells expressing a Dox-inducible random shRNA (shRandom) sequence (Fig. 4C). Next, clonogenic growth was assessed upon Dox-induced Ezh2 knockdown alone or in combination with the ATM inhibitor AZD1390 (Fig. 4D-E). In BRCA1-deficient cells, Ezh2 knockdown combined with AZD1390 induced 64\% reduction in clonogenic growth compared to vehicle control (KB1P-G3-shEzh2: $p<0.0001)$, while shRandom-expression in combination with AZD1390 had no effect (Fig. 4D-E, upper two panels). In BRCA1-proficient cells, a mild effect was observed upon Ezh2 knockdown in combination with AZD1390, however, this effect was not statistically significant (KP3.33-shEzh2: $p=0.12$; Fig. 4D-E, lower two panels).

\section{Combined EZH2/ATM inhibition induces genotoxic stress}

We hypothesized that the observed increase in apoptotic cell death in BRCA1-deficient cells by combined EZH2/ATM inhibition was driven by higher levels of DNA damage due to repressed DSB repair efficiency. We used immunofluorescence staining and high-throughput microscopy of phosphorylated nuclear histone $\mathrm{H} 2 \mathrm{AX}(\mathrm{yH} 2 \mathrm{AX}$ ) foci formation to assess the level of DNA damage and repair ability in BRCA1deficient and BRCA1-proficient mouse mammary tumor cells treated with GSK126 and AZD1390 (Fig. 5AB). The number of $\mathrm{yH} 2 \mathrm{AX}$ foci per cell, which correspond with sites of DSBs, was highest in KB1P-G3 and 
KB1P-B11 cells treated with combined GSK126/AZD1390, showing a 2- to 3-fold increase, compared to vehicle control or single agent treatment (Fig. 5C). Cisplatin served as a positive control and brought about a 2- to 3-fold increased number of $\mathrm{yH} 2 \mathrm{AX}$ foci per cell as a result of DNA adduct formation. As expected, the number of detected cells per field was lowest in GSK126/AZD1390 and cisplatin-treated BRCA1-deficient cells (Supplementary Fig. S4). These results indicate that combined EZH2/ATM inhibition induces increased DNA damage in BRCA1-deficient mouse mammary tumor cells.

\section{Synergy of combined EZH2/ATM inhibition facilitates sensitivity in BRCA1-deficient mouse mammary tumor allografts}

To test the effect of combined EZH2/ATM inhibition in vivo, mice bearing tumor fragments derived from KB1P donor mice (Fig. 6A) were treated with vehicle, GSK126 $(150 \mathrm{mg} / \mathrm{kg}$, by daily intraperitoneal injection), AZD1390 (20 mg/kg, by oral gavage twice daily, 5 days on, 2 days off) or the combination of GSK126 and AZD1390 for 28 consecutive days. As a result of the treatment, progression-free survival was significantly increased by single agent treatment using GSK126 compared to vehicle control $(p=0.002)$, as wells as with AZD1390 treatment compared to vehicle control $(p=0.038)$. The longest progression-free survival was observed in the GSK126/AZD1390 combination therapy cohort, which was significantly increased compared to vehicle $(p=0.0006)$ and to single agent treatment with AZD1390 $(p=0.048)$ (Fig. 6B). Immunohistochemistry analysis of GSK126 and AZD1390 treated tumors confirmed effective target inhibition by reduced H3K27me3 and by phosphorylated ATM levels, respectively (Fig. $6 \mathrm{C})$. The effects of the double agent treatment in vivo recapitulate and confirm our in vitro findings that combined treatment with GSK126 and AZD1390 has a synergistic effect and confers sensitivity in BRCA1-deficient mammary tumors.

\section{Discussion}

The identification of targeted treatment strategies for BRCA1-deficient breast cancer is a current emphasis of preclinical research and clinical practice. Using a high-throughput drug screen, we identified synergy between inhibition of EZH2 and ATM with specific cytotoxicity in BRCA1-deficient tumor cells, thereby providing a molecularly rationalized approach that could guide clinical investigations of a combined therapy with EZH2 and ATM inhibitors in BRCA1-deficient breast cancer. The synergy of a combined EZH2/ATM inhibition in BRCA1-deficient tumor cells could be demonstrated by using several small molecule inhibitors of EZH2 (GSK126, ZLD1039) and ATM (AZD1390, KU60019) that are currently at various stages of preclinical development. Targeting ATM signaling is currently evaluated in HRdeficient breast cancer in several phase I and II trials $(34,38)$, corroborating the clinical significance of our approach. The synergistic cytotoxic effect of combined EZH2/ATM inhibition could further be confirmed by genetic downregulation of $E Z H 2$ in combination with pharmacological ATM inhibition. Our in vivo study shows that the combined treatment with GSK126 and AZD1390 confers stronger anti-tumor activity than either inhibitor alone in BRCA1-deficient mammary tumors in mice, although the effect on progression-free survival was not strong. 
GSK126, one of the first selective EZH2 inhibitors, was extensively tested in DLBCL lymphoma cell lines leading to significant growth inhibition and increased apoptotic rate with maximal potency after 2 days (39). GSK126 administration in preclinical xenograft models showed tumor stasis with $50 \mathrm{mg} / \mathrm{kg}$ once daily, and even complete tumor eradication with $150 \mathrm{mg} / \mathrm{kg}$ (39). Single GSK126 treatment of the TNBC cell line MDA-MB-231 induced only modest inhibition of cell survival (concentrations up to $8 \mu \mathrm{M}$ ), while the combination of GSK126 and gefitinib was synergistic on apoptosis-mediated cell death (40). Phase I clinical trials with single GSK126 treatment in non-hodgkin-lymphoma and multiple myeloma had been discontinued due to insufficient therapeutic activity (ClinicalTrials.gov: NCT02082977) (41). However, novel treatment regimens combining GSK126 with other compounds targeting key signaling pathways or administering GSK126 in cancers harboring synthetic lethal mutations are currently under preclinical investigation and could be evaluated in future clinical trials $(21,42,43)$.

The ATM kinase inhibitor AZD1390 has been tested in preclinical models of glioblastoma multiforme (GBM) and lung cancer as sensitizer to radiotherapy (30). Interestingly, AZD1390 preferentially radiosensitized p53-deficient GBM cells. P53-deficiency disabled GBM cells to induce cell cycle arrest upon radiation-induced DNA damage, which was further exacerbated by AZD1390-mediated ATM inhibition and subsequent failure of DNA damage repair leading to cell death (30). In our in vitro and in vivo models of BRCA1/p53 double-deficient mammary tumors, reflecting the mutational spectrum of human BRCA1-mutant breast cancer, the cellular consequences induced by combined EZH2/ATM inhibition could follow a similar mechanism.

Our data suggest that inhibition of EZH2 methyltransferase activity sensitizes BRCA1-deficient breast cancer cells to ATM inhibition leading to increased yH2AX formation and enhanced apoptosis-mediated cell death (Fig. 7). Since BRCA1-deficient cells experience higher levels of DNA damage due to lower DSB repair efficiency, we assume that those cells display an increased dependence on ATM function for survival. In ovarian cancer cells, EZH2 inhibition has been shown to induce ATM overactivity and cell cycle checkpoint activation (44). The combined inhibition of EZH2 and ATM could provoke an exacerbation of the intrinsic defect of HR-mediated DSB repair leading to apoptosis in BRCA1-deficient cells but not in BRCA1-proficient cells. TNBC is an immunogenic tumor, however, BRCA1 mutation status has not yet been proven as predictive marker for immune checkpoint inhibition $(45,46)$. The ability of combined EZH2/ATM inhibition to propagate DNA damage in BRCA1-deficient breast cancer could enhance the response to immuno-oncological treatment.

EZH2 can affect the cellular response to DNA damage in multiple ways and its precise role remains unclear. It is known that EZH2 confers gene silencing through $\mathrm{H} 3 \mathrm{~K} 27 \mathrm{me} 3$ methylation and chromatin condensation thereby also regulating sensitivity to DNA damage $(47,48)$. Inhibition of EZH2 facilitated chromatin relaxation and was shown to induce increased susceptibility to DNA damage in an ATMdependent manner (48-50). Furthermore, EZH2 depletion was shown to confer replicative stress $(50,51)$. Cancer cells harboring high levels of replication stress are more likely to rely on the ATR/CHK1 pathway for survival (52-54). By contrast, the cellular response to DSBs mainly depends on ATM/CHK2 signaling $(55,56)$. Genetic and pharmacological EZH2 inhibition was shown to diminish DNA damage- 
induced CHK1 phosphorylation, without affecting CHK2 phosphorylation, thereby inducing DNA damageinduced apoptosis (57). This is in line with a report where CHK1 inhibition has been found to be particularly effective against TNBC (58). Increased ATM dependence could thus be due to GSK126induced inhibition of the ATR/CHK1 axis, driving cells to rely on the ATM-mediated DNA damage response. By inhibiting ATM in BRCA1/p53 double-deficient tumor cells, activation of cell cycle checkpoint and DNA damage pathways could be abrogated leading to cell cycle progression, exacerbating the level of genetic lesions, and subsequently leading to apoptosis. Furthermore, synthetic lethality between genes of the Fanconi anemia/BRCA pathway and ATM inhibition were shown to induce toxic levels of NHEJ leading to genomic rearrangements (59), suggesting that toxic NHEJ levels might play a role in the synergistic cytotoxicity between ATM and EZH2 inhibition in BRCA1-deficient cells.

Epigenetic therapies aim at reprogramming the aberrant epigenetic state rather than inducing cytotoxicity. Thus, a major advantage of this approach is that the effective dose level is below the maximal tolerated dose. Therefore, cytotoxic doses of EZH2 inhibitors can be avoided without losing the inhibitory effect on $\mathrm{H} 3 \mathrm{~K} 27$ me3 levels. Using BRCA1 mutation status as predictive biomarker for targeted $\mathrm{EZH} 2$ treatment could circumvent a current major obstacle of rational clinical implementation of EZH2 inhibition since $E Z H 2$ mutation status alone has been shown to be insufficient to stratify patients for therapy $(60,61)$.

\section{Conclusions}

We provide a rationalized approach of a synergistic therapy with EZH2 and ATM inhibition in BRCA1deficient breast cancer that could guide further preclinical and clinical investigations. The identification of novel targeted treatment approaches is of high importance for patients with BRCA1-mutant breast cancer to overcome failure of a chemotherapy or resistance to PARP inhibition. Combined EZH2 and ATM inhibition could also be useful in conjunction with immuno-oncological agents that represent promising therapeutic options for the treatment of TNBC.

\section{Material And Methods}

\section{Integration with TCGA breast cancer patient data}

Breast invasive carcinoma patient data from The Cancer Genome Atlas Breast Cancer (TCGA BRCA) were retrieved using the UCSC Cancer Browser (https://genome-cancer.ucsc.edu/). For graphical view of genomic data, whole-exome sequencing $(n=526)$ data were analyzed using the Xena browser at the cBioPortal (http://www.cbioportal.org). A detailed description of data generation and instructions can be viewed on https://xenabrowser.net (62).

\section{RNA sequencing}

Gene expression was analyzed in KB1P and KP mouse mammary tumors. Illumina TruSeq mRNA libraries were generated and sequenced with $50-65$ base single reads on a HiSeq 2500 using v4 chemistry (Illumina Inc., San Diego). The resulting reads were trimmed using Cutadapt (version 1.15) to remove any 
remaining adapter sequences and to filter reads shorter than $20 \mathrm{bp}$ after trimming to ensure good mappability. The trimmed reads were aligned to the $\mathrm{GRCm} 38$ reference genome using STAR (version 2.6.1a (63)). Gene expression counts were generated by feautureCounts (version 1.5.0-p1 (64)) using genome definitions from Ensembl GRCm38 version 76. Normalized expression values were obtained by correcting for differences in sequencing depth between samples using DESeq median-of-ratios approach (65) and the log-transforming normalized counts. To statistically test the differences between the groups, ANOVA and pairwise t-test with multiple testing correction was used.

\section{Cell lines and culturing}

Murine tumor cell lines were generated from individual tumors arising in female KB1P or KP mice as described previously (29). Established cell lines were cultured at $37^{\circ} \mathrm{C}$ with $5 \%$ carbon dioxide under low oxygen conditions (3\%) in DMEM/F12 medium (Thermo Fisher, Cat\#31331028) supplemented with 10\% FCS, $1 \%$ of Penicillin-Streptomycin (5000 U/mL, Thermo Fisher, Cat\#12140122), $5 \mathrm{mg} / \mathrm{mL}$ insulin (Sigma, Cat\#53003-018), $5 \mathrm{ng} / \mathrm{mL}$ epidermal growth factor (Thermo Fisher, Cat\#16634), and $5 \mathrm{ng} / \mathrm{mL}$ cholera toxin (Gentaur, Cat\#C8052).

KB1P-G3 and KP-3.33 cells stably expressing shEzh2 were cultured in DMEM/F12 medium supplemented with $10 \%$ FCS, $1 \%$ of Penicillin-Streptomycin, $5 \mathrm{mg} / \mathrm{mL}$ insulin, $5 \mathrm{ng} / \mathrm{mL}$ epidermal growth factor, and 5 $\mathrm{ng} / \mathrm{mL}$ cholera toxin. shRNA expression was induced with $100 \mathrm{ng} / \mathrm{ml}$ doxycycline (Dox) (Sigma Aldrich, Cat\#D9891) in culture medium. Medium of uninduced cells was supplemented with the respective volume of PBS.

Human SUM149 cells were cultured in DMEM/F12 medium supplemented with 10\% FCS, $1 \%$ of PenicillinStreptomycin. Human CAL120 cells were cultured in RPMI1640 (Thermo Fisher, Cat\#12633012) supplemented with $10 \%$ FCS, $1 \%$ of Penicillin-Streptomycin.

All cell lines were tested negative for Mycoplasma contamination upon thawing using a PCR Mycoplasma Test Kit (AppliChem, Cat\#A3744).

\section{Pharmacological compounds}

The following compounds were purchased from Selleckchem: AZD1390 (ATM inhibitor, Cat\#S8680), AZD 7762 (CHK1/2 inhibitor, Cat\#S1532), BI2536 (PLK1 inhibitor, Cat\#S1109), BKM120 (PI3K inhibitor, Cat\#S2247), crizotinib (ROS1 inhibitor, Cat\#S1068), dinaciclib (CDK inhibitor, Cat\#S2768), gefitinib (EGFR inhibitor, Cat\#S1025), GSK126 (EZH2 inhibitor, Cat\#S7061), JQ1 (BET inhibitor, Cat\#S7110), KU60019 (ATM inhibitor, Cat\#S1570), KU60648 (DNA-PK inhibitor, Cat\#S8045), LDC67 (CDK9 inhibitor, Cat\#S7461), MK1775 (Wee1 inhibitor, Cat\#S1525), olaparib (PARP inhibitor, Cat\#S1060), palbociclib (CDK4/6 inhibitor, Cat\#S1579), panobinostat (HDAC inhibitor, Cat\#S1030), PF477736 (CHK1 inhibitor, Cat\#S2904), purvalanol A (CDK1/2 inhibitor, Cat\#S7793), RO3306 (CDK1 inhibitor, Cat\#S7747), selisitat (SIRT1 inhibitor, Cat\#S1541), selumetinib (MEK inhibitor, Cat\#S1008), senexin A (CDK8/19 inhibitor, Cat\#S8520), TH287 (MTH1/NUDT1 inhibitor, Cat\#S7631), THZ1 (CDK7 inhibitor, Cat\#S7549), VE822 
(ATR inhibitor, Cat\#S7102), venetoclax (BCL2 inhibitor, Cat\#S8048). NSC663284 (Cdc25 inhibitor, Cat\#383907-43-5) was purchased from Cayman Chemical, PF3644022 (MK2 inhibitor, Cat\#B5549) from ApexBio, ZLD1039 (EZH2 inhibitor, Cat\#AOB9716) from AOBIOUS. All compounds were dissolved in DMSO (Carl Roth, Cat\#A994.2) at a concentration of $10 \mathrm{mM}$. Equal amounts of DMSO added to the cell culture medium served as vehicle control.

\section{Cell viability measurement}

Optimal seeding density per cell line was derived from growth curves performed prior to screening experiments. Cells from subconfluent cell culture dishes were filtered through a cell strainer and viable cells were counted with Countess ${ }^{\mathrm{TM}}$ II FL Automated Cell Counter (Invitrogen) to allow optimal exponential growth (log phase) during the whole experiment. Cells were plated into 384-well plates (500 to 1000 cells/well) in $30 \mu \mathrm{l}$ complete culture medium. The compounds were added after 24 hours by using the TECAN D300e digital dispenser (HP). After 72 hours of treatment, cell viability was assessed by measuring ATP content in each well using CellTiter-Glo Reagent (Promega, Cat\#G7573) 1:1.

Luminescence intensity was measured using a plate reader (Tecan Infinite M1000 Pro) and normalized to intensities of control wells.

\section{Compound synergy screen}

Before screening for drug synergy, single agent effects of all compounds were profiled on the KB1P and $\mathrm{KP}$ mouse mammary tumor cell lines for 10 different concentrations in two-fold serial dilutions, ranging from $20 \mathrm{nM}$ to $20 \mu \mathrm{M}$ and assessed by cell viability measurement using CellTiter-Glo. The concentrationeffect relationship ( $\mathrm{IC}_{50}$ values) was determined by logistic interpolation using GraphPad Prism. See Supplementary Table $\mathrm{S} 1$ for $\mathrm{IC}_{50}$ values.

Next, in a pre-screen for drug synergy, GSK126 was tested against each compound in two-fold serial dilutions to determine the optimal drug concentrations for synergy analysis using a $6 \times 6$ matrix for drug combinations ( 5 concentrations for each compound and DMSO control). For the main synergistic combination screen, 5 representative concentrations titrated around the previous determined $\mathrm{IC}_{50}$ values were used for each compound. The compounds were then profiled in combination with GSK126 using the 6x6 matrix layout, and cells were treated as described above See Supplementary Table S1 for compound concentrations. Cell viability was detected after 72 hours using CellTiter-Glo Reagent. See Supplementary Table S2 for normalized cell viability data used for calculation of synergy scores.

\section{Analysis of synergy}

Analysis of synergy scores was determined using the Bliss independence model calculating the difference between observed and expected compound effects (66) as described before (67). Briefly, single agent effects of compounds $A$ and $B\left(a^{A}, a^{B}\right)$ at concentrations $C x^{A}$ and $C y^{B}$ were used to calculate the expected effect for additive compound interactions $\left(a_{\text {exp }}=a^{A}+a^{B}-a^{A} * a^{B}\right)$, and subsequently compared to the effect observed under combination of both compounds $\left(a_{o b s}\right)$. The delta score $\left(\Delta a=a_{o b s}-a_{\text {exp }}\right)$ 
calculated as the difference between observed and expected effects over the full dose-response matrix characterizes the synergistic effect. A score of $>15$ was used as threshold indicating a synergistic compound interaction (67). Synergistic effects were visualized using the web-application tool SynergyFinder version 2.0 (32).

\section{Clonogenic survival assay and crystal violet staining}

Cells were seeded at a density of 25000 cells per well into 6-well cell culture plates. After overnight incubation, cells were continuously treated with GSK126 $(7.5 \mu \mathrm{M})$ or with AZD1390 $(2 \mu \mathrm{M})$ alone or in combination for 7 days. DMSO was used as vehicle control.

KB1P-G3 and KP-3.33 cells stably expressing shEzh2 or shRandom were seeded into 10-cm cell culture dishes in the appropriate cell culture media 24 hours before treatment. To induce Ezh2 knockdown, cells were continuously treated with Dox $(100 \mathrm{ng} / \mu \mathrm{l})$ for 7 days. For the colony formation assay, cells treated with Dox for 7 days were replated at 10000 cells per well into 12-well cell culture plates and cultivated in Dox for the duration of the entire experiment. Twenty-four hours after replating, cells were exposed to AZD1390 $(2 \mu \mathrm{M})$ and incubated for 7 days in Dox-supplemented culture medium. Uninduced (PBS) and DMSO-treated cells were used as negative control.

After the treatment period, colonies were fixed with methanol on ice, stained with $0.5 \%$ crystal violet (Sigma Aldrich, Cat\#HT90132) and imaged using Carl Zeiss Stemi 2000-C Stereo microscope equipped with a CCD camera (Zeiss) at $0.65 x$ magnification. Colonies were counted on whole surface area using the Clono-counter software and values were normalized to control wells (68).

\section{Real-time cell proliferation assay}

Cells were plated into 384-well plates (500 cells/well) in $30 \mu \mathrm{l} \mathrm{complete} \mathrm{culture} \mathrm{media.} \mathrm{After} \mathrm{overnight}$ incubation, cells were treated with GSK126 $(7.5 \mu \mathrm{M})$, AZD1390 $(2 \mu \mathrm{M})$ or the combination of both. DMSO served as vehicle control. Cells were allowed to grow for 120 hours. Phase-contrast images were automatically acquired by IncuCyte FLR (Essen Bioscience) from the incubator at 4-hour intervals. Proliferation was monitored by analyzing the occupied area (\% confluence) of cell images over time by IncuCyte software (Essen Bioscience).

\section{Cell lysis and immunoblotting}

Whole-cell lysates were prepared in RIPA lysis buffer (50 mM Tris-HCl pH 7.5, $100 \mathrm{mM} \mathrm{NaCl}, 0.1 \%$ SDS, $0.5 \%$ Sodium deoxycholate) supplemented with $1 \mathrm{mM}$ PMSF, $1 \%$ SDS, and phosphatase inhibitors (1x PhosphoSTOP Phosphatase Inhibitor Cocktail, Roche Diagnostics, Cat\#4906845001). Samples were lysed on ice for $20 \mathrm{~min}$, sheared by passing cells through a G25 needle (B. Braun, Hypodermic NeedlePro $\AA$, Cat\#4658304), cleared by centrifugation (14000 x g, $15 \mathrm{~min}$ ), and quantified using Pierce ${ }^{\mathrm{TM}}$ Bradford protein assay kit (Thermo Scientific, Cat\#23200). Lysates were boiled 5 min at $95^{\circ} \mathrm{C}$ with $6 \mathrm{x}$ reducing Laemmli buffer (0.12M Tris pH 6.8, 47\% glycerol, $12 \%$ SDS, $0.6 \mathrm{M}$ DTT, $0.06 \%$ bromophenol 
blue). Samples were separated on a polyacrylamide gel and transferred to PVDF membranes using the mini wet/tank blotting system (Bio-Rad). After blocking with 5\% BSA in Tween-20/PBS, membranes were probed with primary antibodies prepared in blocking solution overnight at $4^{\circ} \mathrm{C}$ on a roller, followed by incubation with horseradish peroxidase-conjugated secondary antibody in blocking solution for $1 \mathrm{~h}$ at room temperature and ECL detection (Thermo Fisher, Cat\#34096) by the ChemiDoc XRS+ system (BioRad). Primary and secondary antibodies used for Western blotting are listed in Supplementary Table S1. Quantitative analysis of protein expression relative to GAPDH was done using Image Lab software (BioRad).

\section{Analysis of apoptosis by flow cytometry}

Cell lines ( 150000 cells/well) were seeded into 6-well plates at 40-60\% confluency. After overnight incubation, medium was replaced with growth medium containing single inhibitors GSK126 $(7.5 \mu \mathrm{M})$ or AZD1390 $(2 \mu \mathrm{M})$ or in combination for 48 hours. Culture medium was collected, cells were trypsinized, washed with ice-cold PBS, and incubated with Annexin-V (BD Bioscience, Cat\#556420) and propidium iodide (PI, Carl Roth, Cat\#CN74, $5 \mu \mathrm{g} / \mathrm{ml})$ in antibody binding buffer $(2.5 \mathrm{mM} \mathrm{CaCl} 2,10 \mathrm{mM} \mathrm{HEPES}(\mathrm{pH}$ 7.4), $140 \mathrm{mM} \mathrm{NaCl}, 20 \%$ accutase solution (Sigma Aldrich, Cat\#A6964), 70\% PBS). Annexin-V/propidium iodide staining was detected by flow cytometry (Beckman Coulter, Gallios Flow Cytometer). The fraction of apoptotic cells was quantified as the Annexin- $\mathrm{V}, \mathrm{PI}$, and double positive stained populations using the Kaluza analysis software (Beckman Coulter).

\section{Immunofluorescence staining and high-throughput microscopy}

Cells (5000 cells/well) were seeded into 96-well imaging plates (Greiner Bio-One, $\mu$ clear, Cat\#655090). After overnight incubation, cells were treated with single or combined agents of GSK126 $(7.5 \mu \mathrm{M})$ and AZD1390 $(2 \mu \mathrm{M})$. For pre-extraction of nonchromatin-bound proteins, after 48 hours of treatment cells were incubated with sucrose buffer ( $25 \mathrm{mM}$ HEPES pH 7.5, $50 \mathrm{mM} \mathrm{NaCl}, 1 \mathrm{mM}$ EDTA, $3 \mathrm{mM} \mathrm{MgCl}$, 300 $\mathrm{mM}$ sucrose, $0.5 \%$ TritonX-100) for $2 \mathrm{~min}$, followed by fixation in $4 \%$ paraformaldehyde for $10 \mathrm{~min}$ at RT. After 1 hour of blocking (5\% BSA, 2\% normal goat serum, $0.1 \%$ TritonX-100, 0.05\% Tween-20) at RT, cells were incubated with primary and secondary antibodies as listed in Supplementary Table S1. After three final washes, cells were covered with PBS and stored at $4^{\circ} \mathrm{C}$ until scanning. Quantitative high-throughput microscopy was performed similarly as described before (67). We used a Thermo Fisher Cellomics CellInsight CX7 LZR High Content Analysis (HCA) Platform with laser light source to scan stained cell models in 96-well imaging plates. $2 \times 2$ binned images ( $1104 \times 1104$ pixels) were acquired with a $20 \times(0.4$ NA) Achroplan objective using the laser-based autofocus and analyzed using the Spotdetector V4.1 Bioapplication of the Cellomics software package (Version 6.6.2, Built 8533). Cell nuclei were identified by Hoechst 34580 staining in background corrected images (3D surface fitting) according to the object identification parameters size: $100-1500 \mu \mathrm{m}^{2}$, ratio of perimeter squared to $4 \pi$ area: $1-5$, length-to-width ratio: $1-5$, average intensity: 500-8000, total intensity: $2 \times 105-5 \times 107$. Foci were identified within the nuclear region using the Box method with a value of 3 . Object selection parameters for foci were $1-30 \mu \mathrm{m}^{2}$, ratio 
of perimeter squared to $4 \pi$ area: $1-5$, length-to-width ratio: $1-5$, average intensity: $500-16000$, total intensity: 3×102-1×106.

\section{Orthotopic tumor transplantation and in vivo drug intervention study}

This study is compliant with all relevant ethical regulations regarding animal research. All animal experiments were approved by the Animal Ethics Committee of The Netherlands Cancer Institute (Amsterdam, the Netherlands) and performed in accordance with the Dutch Act on Animal Experimentation. Generation of conditional K14cre;Brca $7^{\mathrm{F} / \mathrm{F}}$; Trp53 $5 / \mathrm{F}$ (KB1P) and $\mathrm{K} 14 \mathrm{cre}$, $\operatorname{Trp} 53^{\mathrm{F} / \mathrm{F}}(\mathrm{KP})$ breast cancer mouse models were described previously $(69,70)$. Orthotopic transplantations, tumor monitoring, and tissue sampling were performed as described before (71). Briefly, donor KB1P tumor fragments were transplanted into the $4^{\text {th }}$ mammary fat pad of 8-weeks old FVB females and treatments began upon reaching tumor outgrowth of approximately $100 \mathrm{~mm}^{3}(100 \%)$. Maximal tolerable dose (MTD) for the combined therapy with GSK126 and AZD1390 was determined prior to the intervention study using FVB females. GSK126 (Syncom) was reconstituted in 20\% Captisol (CyDex Pharmaceuticals) and brought to a $\mathrm{pH}$ of 4.5 with $10 \mathrm{M}$ potassium hydroxide, to create a working stock of $15 \mathrm{mg} / \mathrm{mL}$. AZD1390 (Selleckchem) was dissolved at $40 \mathrm{mg} / \mathrm{ml}$ in ethanol and then dropwise added to $0.5 \%(\mathrm{w} / \mathrm{v})$ HPMC, $0.1 \%(\mathrm{w} / \mathrm{v})$ Tween-80 to achieve a final concentration of $2 \mathrm{mg} / \mathrm{ml}$. Tumorbearing mice were blindly randomized into four treatment groups and treated with vehicle (daily intraperitoneal injection), GSK126 (150 mg/kg, daily intraperitoneal injection), AZD1390 (bi-daily 20 $\mathrm{mg} / \mathrm{kg}$, by oral gavage for 5 days on, 2 days off) or a combination of GSK126 and AZD1390. Animals were treated for 28 consecutive days and mammary tumor volume $(\mathrm{mm})$ was quantified by caliper measurements using the following formula: $0.5 \times$ length $\times$ width $^{2}$. For a better comparability, the tumor volume at day $x$ was normalized to the initial tumor volume at treatment start (day 0 ) and defined as relative tumor volume (RTV). The endpoint of this study was reached when tumor size was 10 times the RTV, defined as progression-free survival (PFS). Animals were euthanized by $\mathrm{CO}_{2}$ when tumors extended a volume of $1500 \mathrm{~mm}^{3}$ or when severe side effects were observed. All procedures were carried out by animal technicians in a blinded fashion.

\section{In vivo target inhibition by GSK126 and AZD1390}

A small cohort of mice $(n=12)$ was sacrificed after 7 days of single agent or combined treatment with GSK126 and AZD1390, and KB1P tumors were harvested for immunohistochemistry analysis (IHC). IHC staining of EZH2, H3K27me3, and phosphorylated ATM was performed using formalin-fixed paraffinembedded tumor tissue. The following monoclonal antibodies were used for immunohistochemistry: EZH2 (Rb, Cell signaling, Cat\#5246, 1:200), H3K27me3 (Rb, Abcam, Cat\#ab6002, 1:100) and phosphorylated ATM (phospho S1981, Rb, Abcam, Cat\#ab81292, 1:400) overnight at $4^{\circ} \mathrm{C}$. These antibodies were extensively tested for target specificity (14). All slides were digitally processed using the Aperio ScanScope (Aperio, Vista, CA, USA) and captured using ImageScope software version 12.0.0 (Aperio). 


\section{Statistical analysis}

Statistical analysis was performed using GraphPad Prism Version 8 and 9. Mann-Whitney U test, one-way ANOVA with Tukey multiple comparison testing or Kaplan-Meier survival testing with log-rank comparison were used as indicated in the figure legends. P-values below 0.05 were considered statistically significant. Significance levels are indicated as: ${ }^{*}-p \leq 0.05 ;{ }^{* \star}-p \leq 0.01 ; * \star * p \leq 0.001$; nonsignificant levels are not labeled. Number of experimental replicates are indicated in the figure legends.

\section{Abbreviation}

ATM: Ataxia telangiectasia mutated; DSB: Double strand break; EZH2: Enhancer of zeste homolog 2; gBRCA: Germline BRCA1/2 mutation-associated breast cancer; HR: Homologous recombination; NHEJ: Non-homologous end joining; TCGA BRCA: The Cancer Genome Atlas Breast Invasive Carcinoma; TNBC: Triple-negative breast cancer.

\section{Declarations}

\section{Availability of data and materials}

All data generated or analyzed during this study are included in the published article and its supplementary information.

\section{Ethical Approval and Consent to participate}

This study is compliant with all relevant ethical regulations regarding animal research. All animal experiments were approved by the Animal Ethics Committee of The Netherlands Cancer Institute (Amsterdam, the Netherlands) and performed in accordance with the Dutch Act on Animal Experimentation.

\section{Consent for publication}

All authors revised and approved the final manuscript.

\section{Availability of supporting data}

Not applicable.

\section{Competing interests}

H.C.R. received consulting and lecture fees from Abbvie, AstraZeneca, Vertex and Merck. H.C.R. received research funding from Gilead Pharmaceuticals. H.C.R. is a co-founder of CDL Therapeutics GmbH. The remaining authors declare no competing financial interest.

\section{Funding}


This work was funded through the German-Israeli Foundation for Research and Development (I-65412.20-2016 to H.C.R.), the Deutsche Forschungsgemeinschaft (SFB-1399, A01/C02, KFO-286-RP2 and RE 2246/13-1 to H.C.R.), the Deutsche Jose Carreras Leukämie Stiftung (R12/08 to H.C.R.), the Else Kröner-Fresenius Stiftung (EKFS-2014-A06 to H.C.R., 2016_Kolleg.19 to H.C.R., J.P.), the Deutsche Krebshilfe (1117240 and 70113041 to H.C.R.), the German Ministry of Education and Research (BMBF e:Med 01ZX1303A to H.C.R.), the Faculty of Medicine of the University of Cologne (Gerok rotation position to L.R.), the Dutch Cancer Society (NKI 2015-7877 to P.B., J.J., and M.P.G. Vreeswijk [Leiden University Medical Center, Leiden, The Netherlands]), the Lundbeck Foundation (grant R223-2016-956 to J.J., Claus Storgaard Sørensen, and Finn Cilius Nielsen [University of Copenhagen, Copenhagen, Denmark]), the Oncode Institute, which is partly financed by the Dutch Cancer Society, and the Netherlands Organization for Scientific Research (NWO Vici project 91814643 to J.J. and NWO Mosaic program grant 017.008.022 to JJ).

\section{Authors' contributions}

L.R., J.P. and H.C.R. designed the research. L.R., L.B. and J.P. performed the in vitro experiments. C.B., M.v.d.V., and J.J. planned the in vivo experiments. C.B., M.v.d.V., N.P., B.S., and R.d.K.-G. performed the in vivo experiments. P.B., E.M.P., and R.d.B. generated and provided the RNA sequencing data. M.v.L. and G.P. provided and pre-tested the shRNA knock-down constructs. J.I. and T.H. provided analysis of the high-throughput microscopy. L.R. wrote the manuscript and prepared the figures. H.C.R., J.J., and J.P. supervised the research.

\section{Acknowledgements}

We are indebted to our patients, who provided primary material. We thank Alexandra Florin, Marion Müller and Ursula Rommerscheidt-Fuß from the Institute of Pathology, University Hospital Cologne, for their outstanding technical support. We thank the CECAD Imaging Facility and Christian Jüngst for their support in microscopy. We would like to thank the people from the Preclinical Intervention Unit of the Mouse Clinic for Cancer and Ageing (MCCA) at the NKI for their technical support performing the animal experiments, especially Anne Paulien Drenth and Eline van der Burg for technical support.

\section{References}

1. Sorlie T, Tibshirani R, Parker J, Hastie T, Marron JS, Nobel A, et al. Repeated observation of breast tumor subtypes in independent gene expression data sets. Proc Natl Acad Sci U S A. 2003;100(14):841823.

2. Mavaddat N, Barrowdale D, Andrulis IL, Domchek SM, Eccles D, Nevanlinna H, et al. Pathology of breast and ovarian cancers among BRCA1 and BRCA2 mutation carriers: results from the Consortium of Investigators of Modifiers of BRCA1/2 (CIMBA). Cancer Epidemiol Biomarkers Prev. 2012;21(1):134-47. 
3. Telli ML, Timms KM, Reid J, Hennessy B, Mills GB, Jensen KC, et al. Homologous Recombination Deficiency (HRD) Score Predicts Response to Platinum-Containing Neoadjuvant Chemotherapy in Patients with Triple-Negative Breast Cancer. Clin Cancer Res. 2016;22(15):3764-73.

4. Turner N, Tutt A, Ashworth A. Hallmarks of 'BRCAness' in sporadic cancers. Nat Rev Cancer. 2004;4(10):814-9.

5. Tutt A, Tovey H, Cheang MCU, Kernaghan S, Kilburn L, Gazinska P, et al. Carboplatin in BRCA1/2mutated and triple-negative breast cancer BRCAness subgroups: the TNT Trial. Nat Med. 2018;24(5):62837.

6. Poggio F, Bruzzone M, Ceppi M, Ponde NF, La Valle G, Del Mastro L, et al. Platinum-based neoadjuvant chemotherapy in triple-negative breast cancer: a systematic review and meta-analysis. Ann Oncol. 2018;29(7):1497-508.

7. Loibl S, Weber KE, Timms KM, Elkin EP, Hahnen E, Fasching PA, et al. Survival analysis of carboplatin added to an anthracycline/taxane-based neoadjuvant chemotherapy and HRD score as predictor of response-final results from GeparSixto. Ann Oncol. 2018;29(12):2341-7.

8. Hahnen E, Lederer B, Hauke J, Loibl S, Krober S, Schneeweiss A, et al. Germline Mutation Status, Pathological Complete Response, and Disease-Free Survival in Triple-Negative Breast Cancer: Secondary Analysis of the GeparSixto Randomized Clinical Trial. JAMA Oncol. 2017;3(10):1378-85.

9. Sikov WM, Berry DA, Perou CM, Singh B, Cirrincione CT, Tolaney SM, et al. Impact of the addition of carboplatin and/or bevacizumab to neoadjuvant once-per-week paclitaxel followed by dose-dense doxorubicin and cyclophosphamide on pathologic complete response rates in stage II to III triple-negative breast cancer: CALGB 40603 (Alliance). J Clin Oncol. 2015;33(1):13-21.

10. Venkitaraman AR. Cancer susceptibility and the functions of BRCA1 and BRCA2. Cell. 2002;108(2):171-82.

11. Hakem R, de la Pompa JL, Mak TW. Developmental studies of Brca1 and Brca2 knock-out mice. J Mammary Gland Biol Neoplasia. 1998;3(4):431-45.

12. Robson M, Im SA, Senkus E, Xu B, Domchek SM, Masuda N, et al. Olaparib for Metastatic Breast Cancer in Patients with a Germline BRCA Mutation. N Engl J Med. 2017;377(6):523-33.

13. Noordermeer SM, van Attikum H. PARP Inhibitor Resistance: A Tug-of-War in BRCA-Mutated Cells. Trends Cell Biol. 2019;29(10):820-34.

14. Puppe J, Opdam M, Schouten PC, Jozwiak K, Lips E, Severson T, et al. EZH2 Is Overexpressed in BRCA1-like Breast Tumors and Predictive for Sensitivity to High-Dose Platinum-Based Chemotherapy. Clin Cancer Res. 2019;25(14):4351-62. 
15. Wang $L$, Zeng $X$, Chen S, Ding L, Zhong J, Zhao JC, et al. BRCA1 is a negative modulator of the PRC2 complex. EMBO J. 2013;32(11):1584-97.

16. Gupta RA, Shah N, Wang KC, Kim J, Horlings HM, Wong DJ, et al. Long non-coding RNA HOTAIR reprograms chromatin state to promote cancer metastasis. Nature. 2010;464(7291):1071-6.

17. Cao R, Wang L, Wang $H$, Xia L, Erdjument-Bromage $H$, Tempst $P$, et al. Role of histone $H 3$ lysine 27 methylation in Polycomb-group silencing. Science. 2002;298(5595):1039-43.

18. Chase A, Cross NC. Aberrations of EZH2 in cancer. Clin Cancer Res. 2011;17(9):2613-8.

19. Puppe J, Drost R, Liu X, Joosse SA, Evers B, Cornelissen-Steijger P, et al. BRCA1-deficient mammary tumor cells are dependent on EZH2 expression and sensitive to Polycomb Repressive Complex 2-inhibitor 3-deazaneplanocin A. Breast Cancer Res. 2009;11(4):R63.

20. Yomtoubian S, Lee SB, Verma A, Izzo F, Markowitz G, Choi H, et al. Inhibition of EZH2 Catalytic Activity Selectively Targets a Metastatic Subpopulation in Triple-Negative Breast Cancer. Cell Rep. 2020;30(3):755-70 e6.

21. Yang H, Cui W, Wang L. Epigenetic synthetic lethality approaches in cancer therapy. Clin Epigenetics. 2019;11(1):136.

22. Hirukawa A, Smith HW, Zuo D, Dufour CR, Savage P, Bertos N, et al. Targeting EZH2 reactivates a breast cancer subtype-specific anti-metastatic transcriptional program. Nat Commun. 2018;9(1):2547.

23. Van der Meulen J, Sanghvi V, Mavrakis K, Durinck K, Fang F, Matthijssens F, et al. The H3K27me3 demethylase UTX is a gender-specific tumor suppressor in T-cell acute lymphoblastic leukemia. Blood. 2015;125(1):13-21.

24. Wilson BG, Wang X, Shen X, McKenna ES, Lemieux ME, Cho YJ, et al. Epigenetic antagonism between polycomb and SWI/SNF complexes during oncogenic transformation. Cancer Cell. 2010;18(4):316-28.

25. Fillmore CM, Xu C, Desai PT, Berry JM, Rowbotham SP, Lin YJ, et al. EZH2 inhibition sensitizes BRG1 and EGFR mutant lung tumours to Topoll inhibitors. Nature. 2015;520(7546):239-42.

26. Bitler BG, Aird KM, Garipov A, Li H, Amatangelo M, Kossenkov AV, et al. Synthetic lethality by targeting EZH2 methyltransferase activity in ARID1A-mutated cancers. Nat Med. 2015;21(3):231-8.

27. Hurvitz SA, Goncalves A, Rugo HS, Lee KH, Fehrenbacher L, Mina LA, et al. Talazoparib in Patients with a Germline BRCA-Mutated Advanced Breast Cancer: Detailed Safety Analyses from the Phase III EMBRACA Trial. Oncologist. 2019. 
28. Moore K, Colombo N, Scambia G, Kim BG, Oaknin A, Friedlander M, et al. Maintenance Olaparib in Patients with Newly Diagnosed Advanced Ovarian Cancer. N Engl J Med. 2018;379(26):2495-505.

29. Silver DP, Dimitrov SD, Feunteun J, Gelman R, Drapkin R, Lu SD, et al. Further evidence for BRCA1 communication with the inactive $X$ chromosome. Cell. 2007;128(5):991-1002.

30. Durant ST, Zheng L, Wang Y, Chen K, Zhang L, Zhang T, et al. The brain-penetrant clinical ATM inhibitor AZD1390 radiosensitizes and improves survival of preclinical brain tumor models. Sci Adv. 2018;4(6):eaat1719.

31. Golding SE, Rosenberg E, Valerie N, Hussaini I, Frigerio M, Cockcroft XF, et al. Improved ATM kinase inhibitor KU-60019 radiosensitizes glioma cells, compromises insulin, AKT and ERK prosurvival signaling, and inhibits migration and invasion. Mol Cancer Ther. 2009;8(10):2894-902.

32. Ianevski A, Giri AK, Aittokallio T. SynergyFinder 2.0: visual analytics of multi-drug combination synergies. Nucleic Acids Res. 2020;48(W1):W488-W93.

33. Blackford AN, Jackson SP. ATM, ATR, and DNA-PK: The Trinity at the Heart of the DNA Damage Response. Mol Cell. 2017;66(6):801-17.

34. Choi M, Kipps T, Kurzrock R. ATM Mutations in Cancer: Therapeutic Implications. Mol Cancer Ther. 2016;15(8):1781-91.

35. Vlot AHC, Aniceto N, Menden MP, Ulrich-Merzenich G, Bender A. Applying synergy metrics to combination screening data: agreements, disagreements and pitfalls. Drug Discov Today. 2019;24(12):2286-98.

36. Song X, Gao T, Wang N, Feng Q, You X, Ye T, et al. Selective inhibition of EZH2 by ZLD1039 blocks H3K27 methylation and leads to potent anti-tumor activity in breast cancer. Sci Rep. 2016;6:20864.

37. Herold MJ, van den Brandt J, Seibler J, Reichardt HM. Inducible and reversible gene silencing by stable integration of an shRNA-encoding lentivirus in transgenic rats. Proc Natl Acad Sci U S A. 2008;105(47):18507-12.

38. DiTullio RA, Jr., Mochan TA, Venere M, Bartkova J, Sehested M, Bartek J, et al. 53BP1 functions in an ATM-dependent checkpoint pathway that is constitutively activated in human cancer. Nat Cell Biol. 2002;4(12):998-1002.

39. McCabe MT, Ott HM, Ganji G, Korenchuk S, Thompson C, Van Aller GS, et al. EZH2 inhibition as a therapeutic strategy for lymphoma with EZH2-activating mutations. Nature. 2012;492(7427):108-12.

40. Yang Y, Zhu F, Wang Q, Ding Y, Ying R, Zeng L. Inhibition of EZH2 and EGFR produces a synergistic effect on cell apoptosis by increasing autophagy in gastric cancer cells. Onco Targets Ther. 
41. Zeng D, Liu M, Pan J. Blocking EZH2 methylation transferase activity by GSK126 decreases stem cell-like myeloma cells. Oncotarget. 2017;8(2):3396-411.

42. Park S, Jo SH, Kim JH, Kim SY, Ha JD, Hwang JY, et al. Combination Treatment with GSK126 and Pomalidomide Induces B-Cell Differentiation in EZH2 Gain-of-Function Mutant Diffuse Large B-Cell Lymphoma. Cancers (Basel). 2020;12(9).

43. Liu S, Rong G, Li X, Geng L, Zeng Z, Jiang D, et al. Diosgenin and GSK126 Produce Synergistic Effects on Epithelial-Mesenchymal Transition in Gastric Cancer Cells by Mediating EZH2 via the Rho/ROCK Signaling Pathway. Onco Targets Ther. 2020;13:5057-67.

44. Naskou J, Beiter Y, van Rensburg R, Honisch E, Rudelius M, Schlensog M, et al. EZH2 Loss Drives Resistance to Carboplatin and Paclitaxel in Serous Ovarian Cancers Expressing ATM. Mol Cancer Res. 2020;18(2):278-86.

45. Voorwerk L, Slagter M, Horlings HM, Sikorska K, van de Vijver KK, de Maaker M, et al. Immune induction strategies in metastatic triple-negative breast cancer to enhance the sensitivity to PD-1 blockade: the TONIC trial. Nat Med. 2019;25(6):920-8.

46. Kraya AA, Maxwell KN, Wubbenhorst B, Wenz BM, Pluta J, Rech AJ, et al. Genomic Signatures Predict the Immunogenicity of BRCA-Deficient Breast Cancer. Clin Cancer Res. 2019;25(14):4363-74.

47. Cann KL, Dellaire G. Heterochromatin and the DNA damage response: the need to relax. Biochem Cell Biol. 2011;89(1):45-60.

48. Kuser-Abali G, Gong L, Yan J, Liu Q, Zeng W, Williamson A, et al. An EZH2-mediated epigenetic mechanism behind p53-dependent tissue sensitivity to DNA damage. Proc Natl Acad Sci U S A. 2018;115(13):3452-7.

49. Xu L, Tang H, Wang K, Zheng Y, Feng J, Dong H, et al. Pharmacological inhibition of EZH2 combined with DNAdamaging agents interferes with the DNA damage response in MM cells. Mol Med Rep. 2019;19(5):4249-55.

50. Ito T, Teo YV, Evans SA, Neretti N, Sedivy JM. Regulation of Cellular Senescence by Polycomb Chromatin Modifiers through Distinct DNA Damage- and Histone Methylation-Dependent Pathways. Cell Rep. 2018;22(13):3480-92.

51. Leon TE, Rapoz-D'Silva T, Bertoli C, Rahman S, Magnussen M, Philip B, et al. EZH2-Deficient Tcell Acute Lymphoblastic Leukemia Is Sensitized to CHK1 Inhibition through Enhanced Replication Stress. Cancer Discov. 2020;10(7):998-1017. 
52. Rundle S, Bradbury A, Drew Y, Curtin NJ. Targeting the ATR-CHK1 Axis in Cancer Therapy. Cancers (Basel). 2017;9(5).

53. Krajewska M, Fehrmann RS, Schoonen PM, Labib S, de Vries EG, Franke L, et al. ATR inhibition preferentially targets homologous recombination-deficient tumor cells. Oncogene. 2015;34(26):3474-81.

54. Minchom A, Aversa C, Lopez J. Dancing with the DNA damage response: next-generation anticancer therapeutic strategies. Ther Adv Med Oncol. 2018;10:1758835918786658.

55. Herlihy AE, de Bruin RA. The Role of the Transcriptional Response to DNA Replication Stress. Genes (Basel). 2017;8(3).

56. Zou Y, Liu Y, Wu X, Shell SM. Functions of human replication protein A (RPA): from DNA replication to DNA damage and stress responses. J Cell Physiol. 2006;208(2):267-73.

57. Wu Z, Lee ST, Qiao Y, Li Z, Lee PL, Lee YJ, et al. Polycomb protein EZH2 regulates cancer cell fate decision in response to DNA damage. Cell Death Differ. 2011;18(11):1771-9.

58. Manic G, Obrist F, Sistigu A, Vitale I. Trial Watch: Targeting ATM-CHK2 and ATR-CHK1 pathways for anticancer therapy. Mol Cell Oncol. 2015;2(4):e1012976.

59. Cai MY, Dunn CE, Chen W, Kochupurakkal BS, Nguyen H, Moreau LA, et al. Cooperation of the ATM and Fanconi Anemia/BRCA Pathways in Double-Strand Break End Resection. Cell Rep. 2020;30(7):2402-15 e5.

60. Bradley WD, Arora S, Busby J, Balasubramanian S, Gehling VS, Nasveschuk CG, et al. EZH2 inhibitor efficacy in non-Hodgkin's lymphoma does not require suppression of H3K27 monomethylation. Chem Biol. 2014;21(11):1463-75.

61. Knutson SK, Warholic NM, Johnston LD, Klaus CR, Wigle TJ, Iwanowicz D, et al. Synergistic AntiTumor Activity of EZH2 Inhibitors and Glucocorticoid Receptor Agonists in Models of Germinal Center Non-Hodgkin Lymphomas. PLoS One. 2014;9(12):e111840.

62. Goldman MJ, Craft B, Hastie M, Repecka K, McDade F, Kamath A, et al. Visualizing and interpreting cancer genomics data via the Xena platform. Nat Biotechnol. 2020;38(6):675-8.

63. Dobin A, Davis CA, Schlesinger F, Drenkow J, Zaleski C, Jha S, et al. STAR: ultrafast universal RNA-seq aligner. Bioinformatics. 2013;29(1):15-21.

64. Liao Y, Smyth GK, Shi W. featureCounts: an efficient general purpose program for assigning sequence reads to genomic features. Bioinformatics. 2014;30(7):923-30.

65. Anders S, Huber W. Differential expression analysis for sequence count data. Genome Biol. 2010;11(10):R106. 
66. BLISS CI. THE TOXICITY OF POISONS APPLIED JOINTLY1. Annals of Applied Biology. 1939;26(3):585-615.

67. Erber J, Steiner JD, Isensee J, Lobbes LA, Toschka A, Beleggia F, et al. Dual Inhibition of GLUT1 and the ATR/CHK1 Kinase Axis Displays Synergistic Cytotoxicity in KRAS-Mutant Cancer Cells. Cancer Res. 2019;79(19):4855-68.

68. Niyazi M, Niyazi I, Belka C. Counting colonies of clonogenic assays by using densitometric software. Radiat Oncol. 2007;2:4.

69. Jonkers J, Meuwissen R, van der Gulden H, Peterse H, van der Valk M, Berns A. Synergistic tumor suppressor activity of BRCA2 and p53 in a conditional mouse model for breast cancer. Nat Genet. 2001;29(4):418-25.

70. Liu X, Holstege $\mathrm{H}$, van der Gulden $\mathrm{H}$, Treur-Mulder M, Zevenhoven J, Velds A, et al. Somatic loss of BRCA1 and $p 53$ in mice induces mammary tumors with features of human BRCA1-mutated basal-like breast cancer. Proc Natl Acad Sci U S A. 2007;104(29):12111-6.

71. Rottenberg S, Jaspers JE, Kersbergen A, van der Burg E, Nygren AO, Zander SA, et al. High sensitivity of BRCA1-deficient mammary tumors to the PARP inhibitor AZD2281 alone and in combination with platinum drugs. Proc Natl Acad Sci U S A. 2008;105(44):17079-84.

\section{Figures}


A

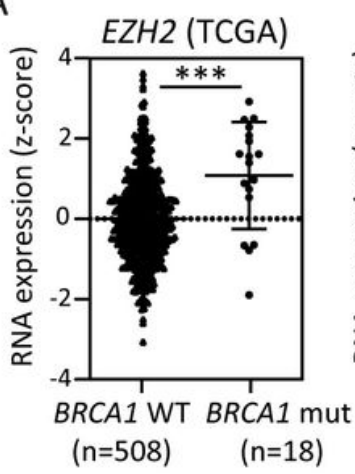

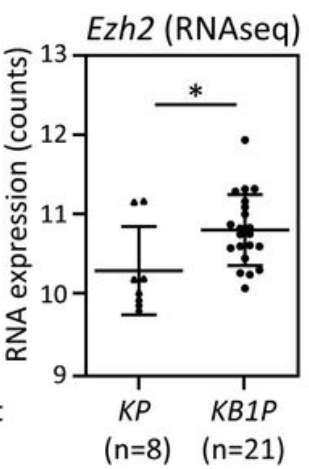

B

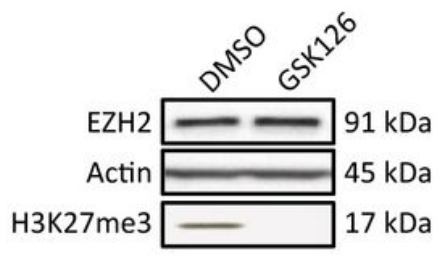

C

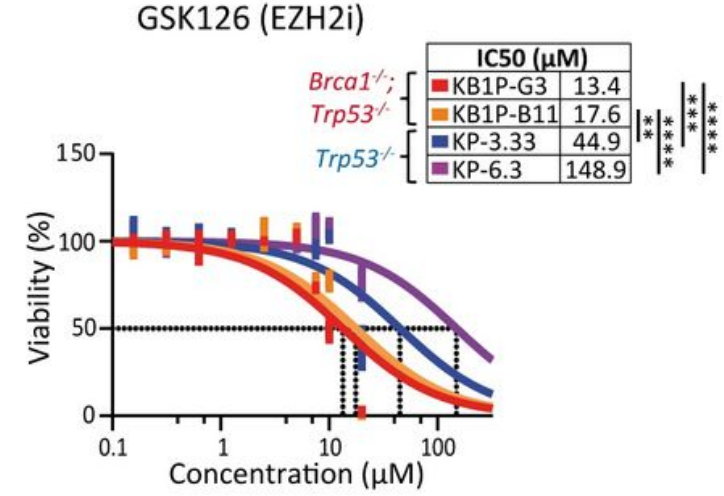

D

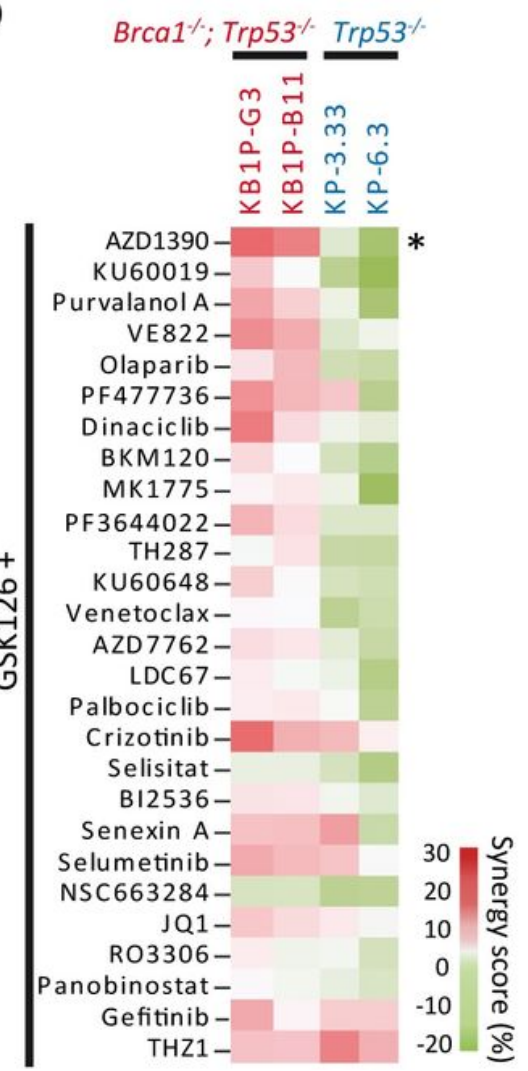

$\mathrm{E}$
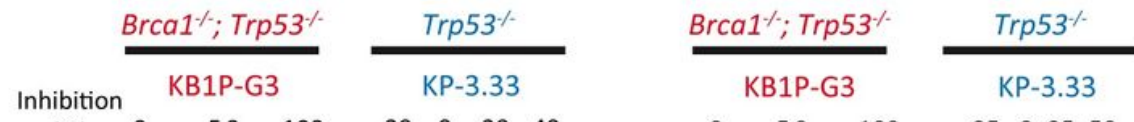

(\%) $\begin{array}{lllllll}0 & 50 & 100 & -20 & 0 & 20 & 40\end{array}$

\begin{tabular}{lllllll}
0 & $50 \quad 100$ & -25 & 0 & $25 \quad 50$ \\
\hline
\end{tabular}
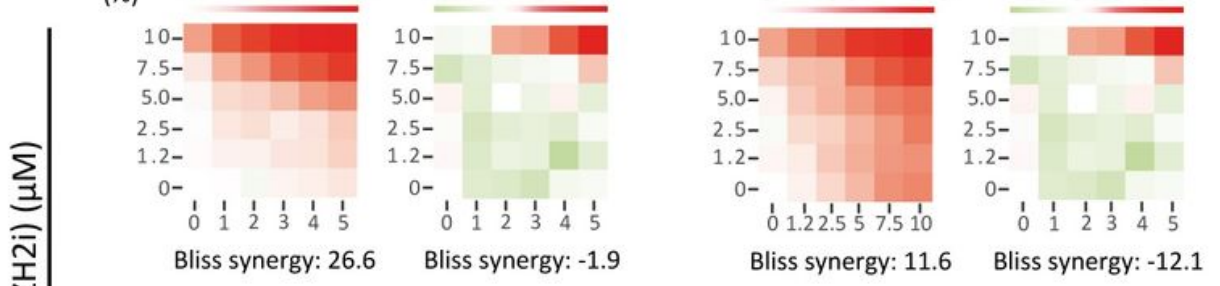

Inhibition KB1P-B11 KP-6.3

$\begin{array}{lllllll}(\%) & 0 & 50 & 100 & -20 & -10 & 0\end{array}$

KB1P-B11

KP-6.3

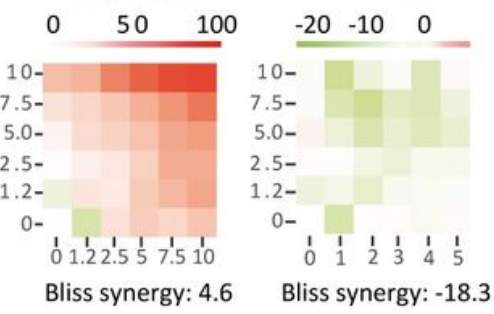

AZD1390 (ATMi) ( $\mu \mathrm{M})$

KU60019 (ATMi) ( $\mu M)$

\section{Figure 1}

Synergistic cytotoxicity screen in combination with GSK126-induced EZH2 inhibition. (A) Increased EZH2 mRNA expression in BRCA1-deficient breast cancer. Left panel: EZH2 expression using the TCGA breast invasive carcinoma (BRCA) whole-exome sequencing data set $(n=826)$. Data are shown as $z$-score transformed in BRCA1-wild type $(n=508)$ versus BRCA1-mutated $(n=18)$ breast tumors. Right panel: Ezh2 expression using RNAseq data from BRCA1-proficient KP $(n=8)$ and BRCA1-deficient KB1P mouse mammary tumors $(n=21)$. Groups were compared using an unpaired Mann-Whitney $U$ test. (B) Target inhibition of H3K27me3 after 72 hours of GSK126 treatment ( $5 \mu \mathrm{M})$ shown by a representative Western blot in BRCA1-deficient KB1P-G3 cells, $\beta$-actin served as loading control. (C) Single agent dose-response curves of GSK126 in four mouse mammary tumor cell lines. Statistical significance was tested by oneway ANOVA with Tukey multiple comparison test. (D) Heatmap showing synergy scores calculated by the Bliss independence model of 27 compounds in combination with GSK126 across four murine mammary 
tumor cell lines. Combinations are arranged by the difference in synergy score between BRCA1-deficient and BRCA1-proficient cell lines from high to low. Cell lines are arranged by the Brca1 mutation status. Concentrations and IC50 values of single compounds can be found in Supplementary Table S1. Asterix indicates the prioritized compound AZD1390. (E) Heatmaps visualized by the web-application tool SynergyFinder showing synergy scores of treatment combinations GSK126/AZD1390 and GSK126/KU60019, respectively. Displayed colors reflect the growth inhibition in percent with red indicating stronger inhibition and green indicating lower inhibition.

A

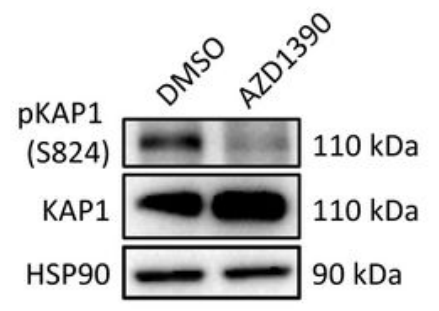

B

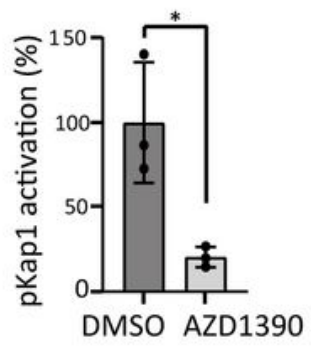

C
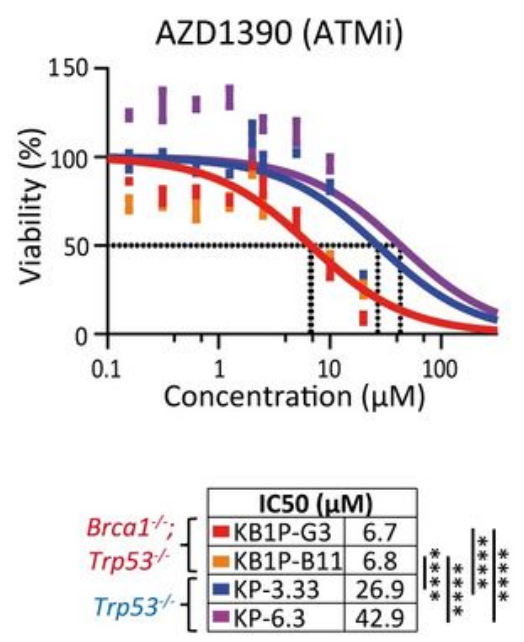

D
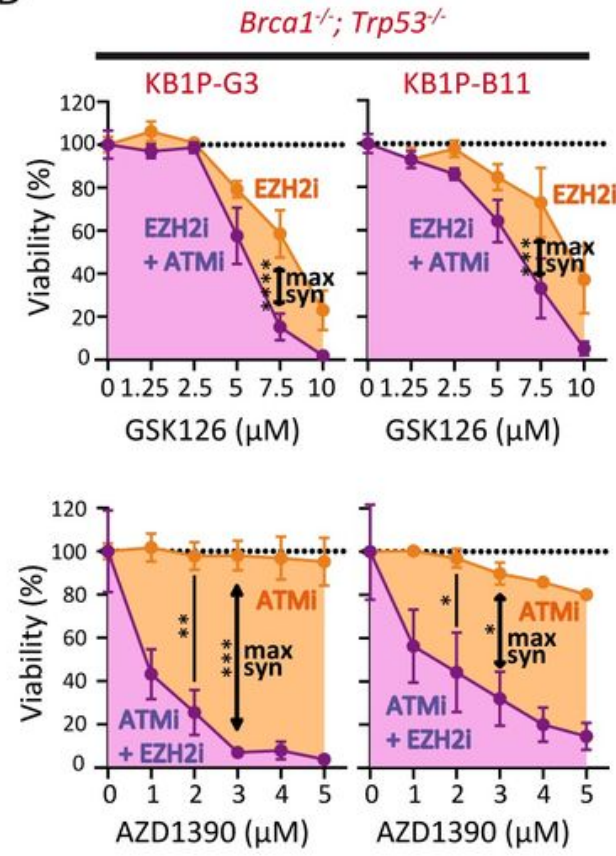

E

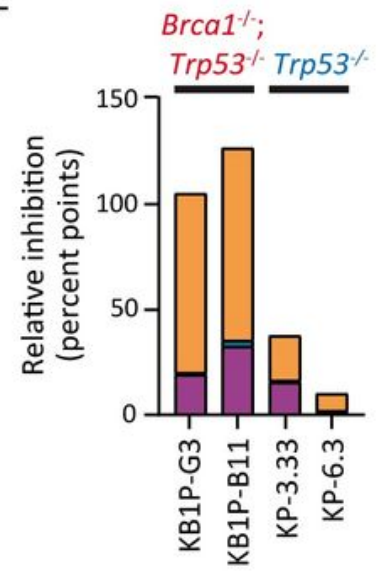

口 GSK126 (7.5 $\mu \mathrm{M})$

口AZD1390 (2 $\mu \mathrm{M})$

口 GSK126 $(7.5 \mu \mathrm{M})+$ AZD1390 (2 $\mu \mathrm{M})$
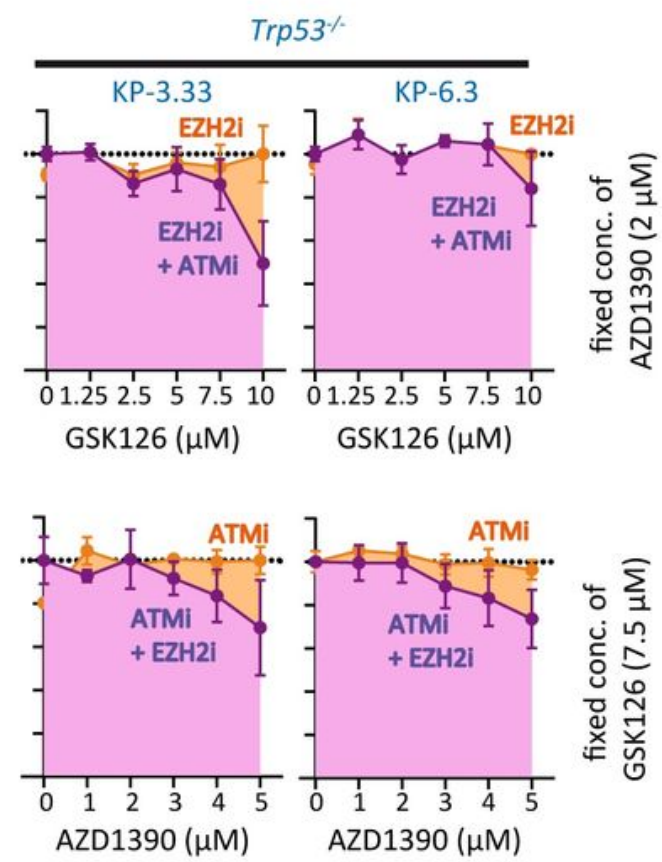

F
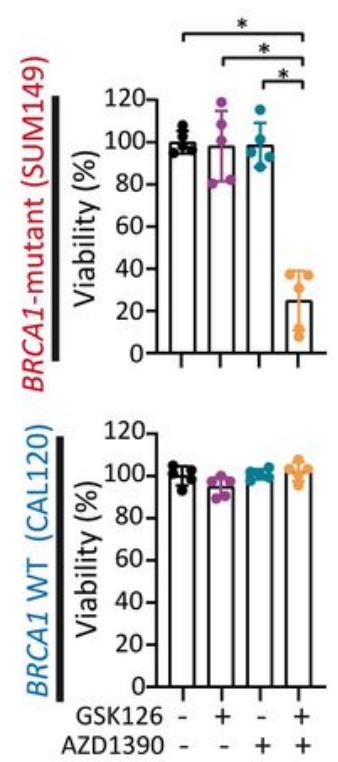

\begin{tabular}{|l|l|}
\hline Synergy score & EZH2i/ATMi \\
\hline
\end{tabular} \begin{tabular}{|l|l|}
\hline BRCA1-mutant & $81.9 \pm 22.5 \%$ \\
\hline
\end{tabular} \begin{tabular}{l|l} 
BRCA1 WT & $-6.0 \pm 5.1 \%$
\end{tabular} 


\section{Figure 2}

Optimization and validation of synergistic effect of EZH2/ATM inhibition. (A) Target inhibition by AZD1390 $(2 \mu \mathrm{M})$ of phosphorylated Kap1 (S824) shown by a representative Western blot in KB1P G3 cells, HSP90 served as loading control. (B) pKap1/Kap1 ratio after densitometric analysis of Western blot bands of three independent experiments. Significance level was assessed by an unpaired t-test. (C) Single agent dose-response curves of AZD1390 in four mouse mammary tumor cell lines. (D) Determination of optimized synergistic compound concentrations for the prioritized drug combination GSK126/AZD1390 resulting in maximal synergistic effects in BRCA1-deficient cells. Upper panel: dose-response curves of increasing concentrations of GSK126 alone (orange) or in combination (purple) with a fixed concentration of AZD1390 ( $2 \mu \mathrm{M})$. Lower panel: dose-response curves of AZD1390 alone (orange) or in combination (purple) with a fixed concentration of GSK126 (7.5 $\mu \mathrm{M})$. Black arrows indicate inhibitor concentrations with maximal synergistic effect. (E) EZH2 inhibition specifically sensitizes BRCA1deficient cells to ATM inhibition. The stacked bar graphs show the relative contribution to inhibition of cell viability by combined treatment with GSK126/AZD1390. (F) Cell viability assay showing increased cytotoxicity using combined EZH2/ATM inhibition in BRCA1-mutant SUM149 (upper panel, Bliss synergy score: $81.9 \pm 22.5 \%$ ) compared to BRCA1-wild type CAL120 (lower panel, synergy score: $-6.0 \pm 5.1 \%$ ) human breast cancer cells. Bars presented as mean \pm SD of five independent experiments. Statistical significance was tested by one-way ANOVA with Tukey multiple comparison test. 
A
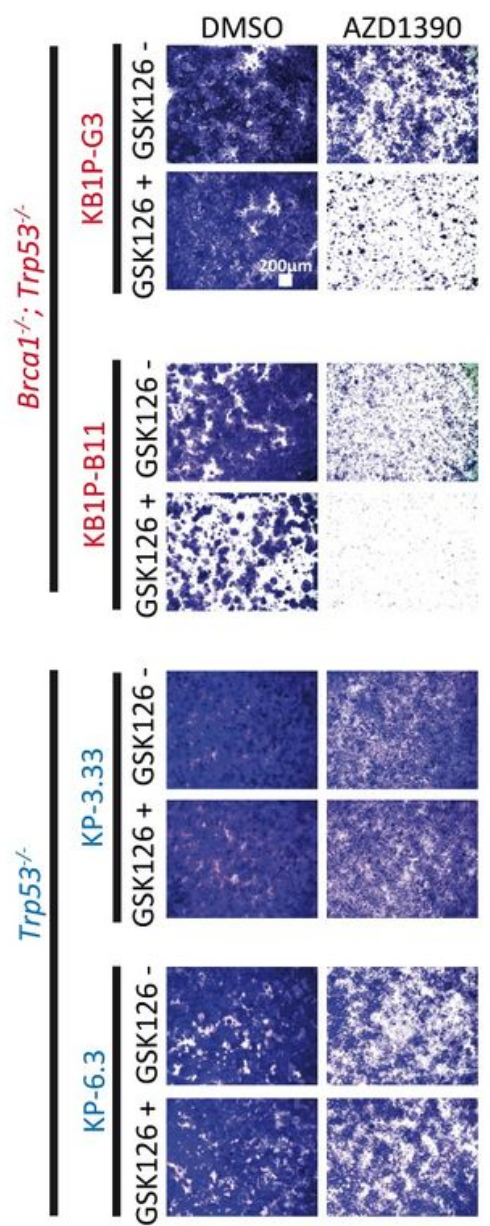

C
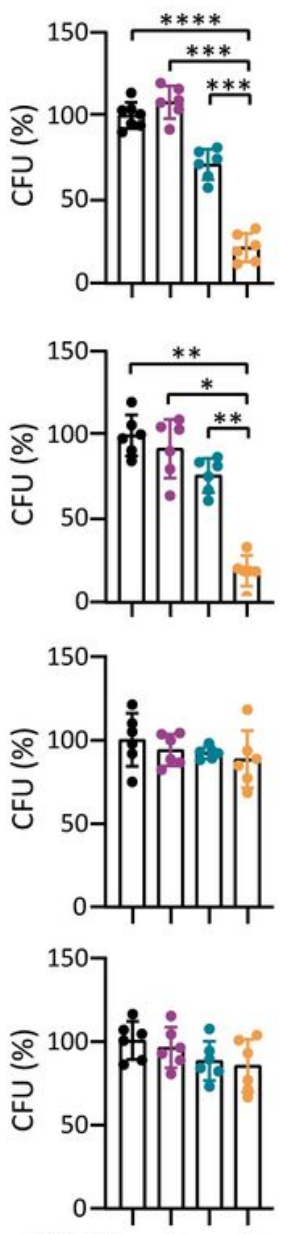

GSK126 - + - + AZD1390 - - + +

\begin{tabular}{|c|c|}
\hline Synergy score & EZH2i/ATMi \\
\hline KB1P-G3 & $55.5 \pm 9.8 \%$ \\
\hline KB1P-B11 & $50.7 \pm 21.9 \%$ \\
\hline KP-3.33 & $-1.6 \pm 20.2 \%$ \\
\hline KP-6.3 & $-1.3 \pm 18.4 \%$ \\
\hline
\end{tabular}
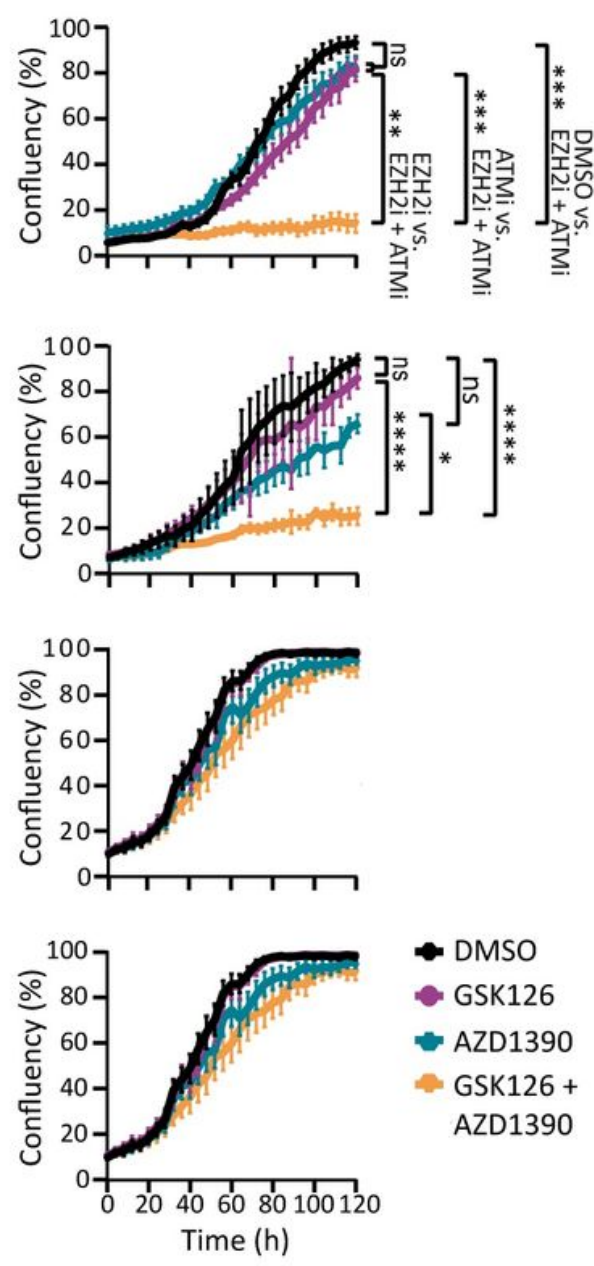

\begin{tabular}{|c|c|}
\hline Synergy score & EZH2i/ATMi \\
\hline KB1P-G3 & $52.0 \pm 8.1 \%$ \\
\hline KB1P-B11 & $32.7 \pm 13.3 \%$ \\
\hline KP-3.33 & $2.4 \pm 8.5 \%$ \\
\hline KP-6.3 & $3.7 \pm 3.3 \%$ \\
\hline
\end{tabular}

D
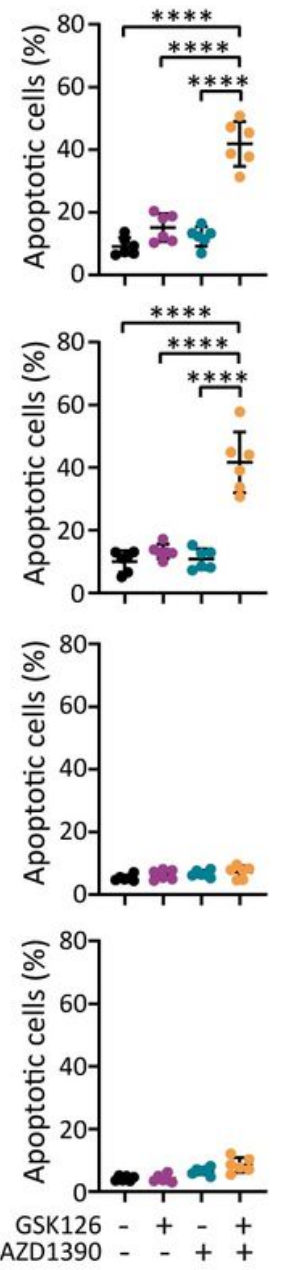

\begin{tabular}{|c|c|}
\hline Synergy score & EZH2i/ATMi \\
\hline KB1P-G3 & $20.8 \pm 10.3 \%$ \\
\hline KB1P-B11 & $22.4 \pm 8.3 \%$ \\
\hline KP-3.33 & $-4.5 \pm 2.4 \%$ \\
\hline KP-6.3 & $-0.4 \pm 1.8 \%$ \\
\hline
\end{tabular}

Figure 3

Functional characterization of combined EZH2/ATM inhibition. (A-B) Colony formation assay in BRCA1deficient (KB1P-G3 and KB1P-B11) and BRCA1-proficient (KP-3.33 and KP-6.3) mouse mammary tumor cells. (A) Representative microscopic images of colony formation at $0.56 x$ magnification. Scale bar 200 $\mu \mathrm{m}$. (B) Quantification of crystal violet positive stained cells is presented as mean \pm SD of six independent experiments. Calculation of drug synergy using the Bliss independence score revealed a mean synergy score of $55.5 \pm 9.8 \%$ in KB1P-G3, $50.7 \pm 21.9 \%$ in KB1P-B11, $-1.6 \pm 20.2 \%$ in KP-3.33, and $-1.3 \pm 18.4 \%$ in KP-6.3 cells. (C) Growth inhibition curves using IncuCyte live cell imaging measured by cell densities on culture plates from at least three independent experiments with each three technical replicates. Bliss synergy score: KB1P-G3: $52.0 \pm$ 8.1\%; KB1P-B11: $32.7 \pm 13.3 \%$; KP-3.33: $2.4 \pm$ 8.5\%; KP6.3: $3.7 \pm 3.3 \%$. (D) Flow cytometric analysis of apoptotic cells measured by Annexin $V$ and propidium iodide double staining of cells treated with GSK126 and AZD1390. Bliss synergy score: KB1P-G3: $20.8 \pm$ 
10.3\%; KB1P-B11: $22.4 \pm 8.3 \%$; KP-3.33: $-4.5 \pm 2.4 \%$; KP-6.3: $-0.4 \pm 1.8 \%$. Significance was tested by oneway ANOVA with Tukey multiple comparison test. CFU: colony forming unit.

A
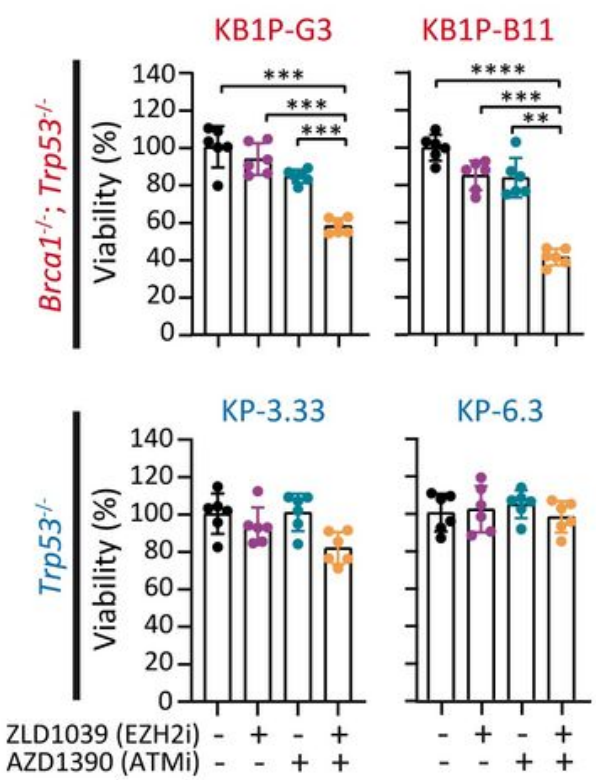

\begin{tabular}{|c|c|}
\hline Synergy score & EZH2i/ATMi \\
\hline KB1P-G3 & $32.2 \pm 13.1 \%$ \\
\hline KB1P-B11 & $36.5 \pm 10.0 \%$ \\
\hline KP-3.33 & $12.3 \pm 9.7 \%$ \\
\hline KP-6.3 & $10.1 \pm 9.7 \%$ \\
\hline
\end{tabular}

B

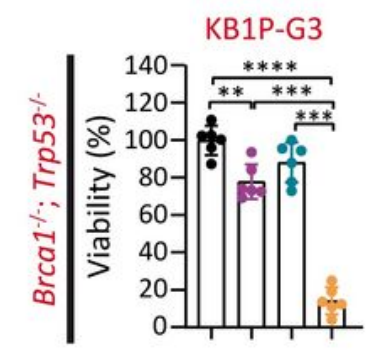

KB1P-B11

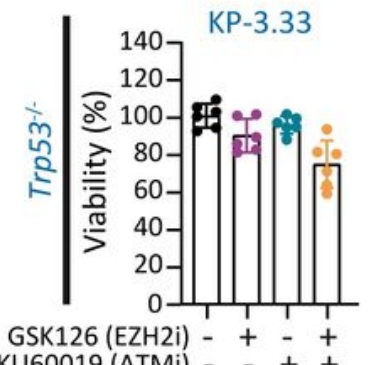

KU60019 (ATMi) - - + +
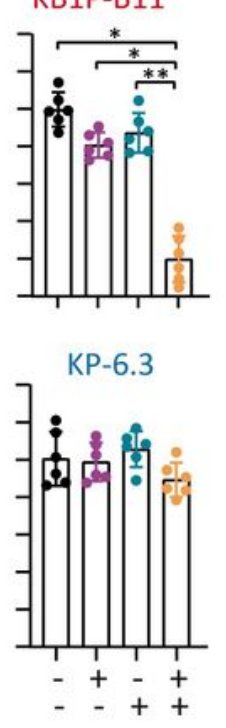

C

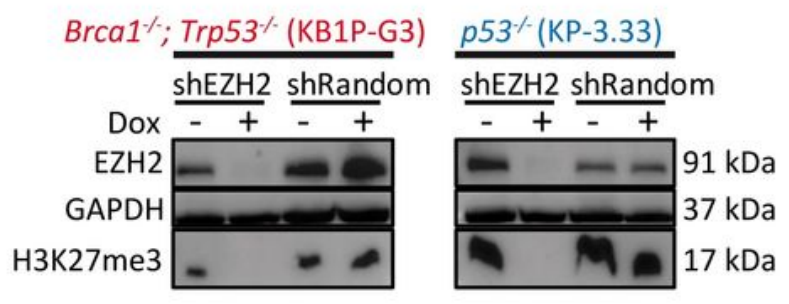

D

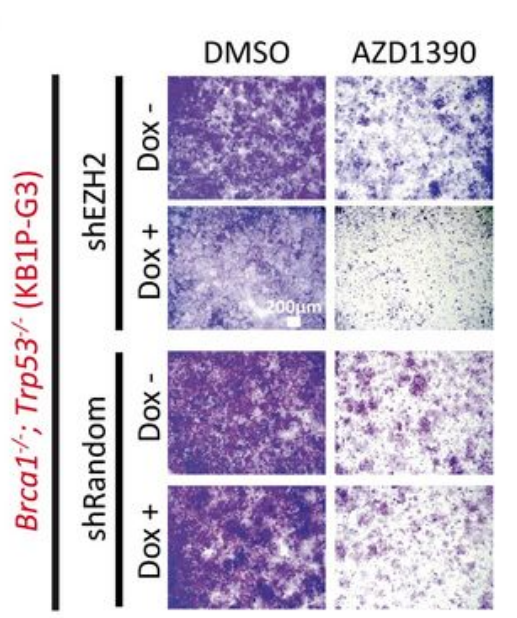

E
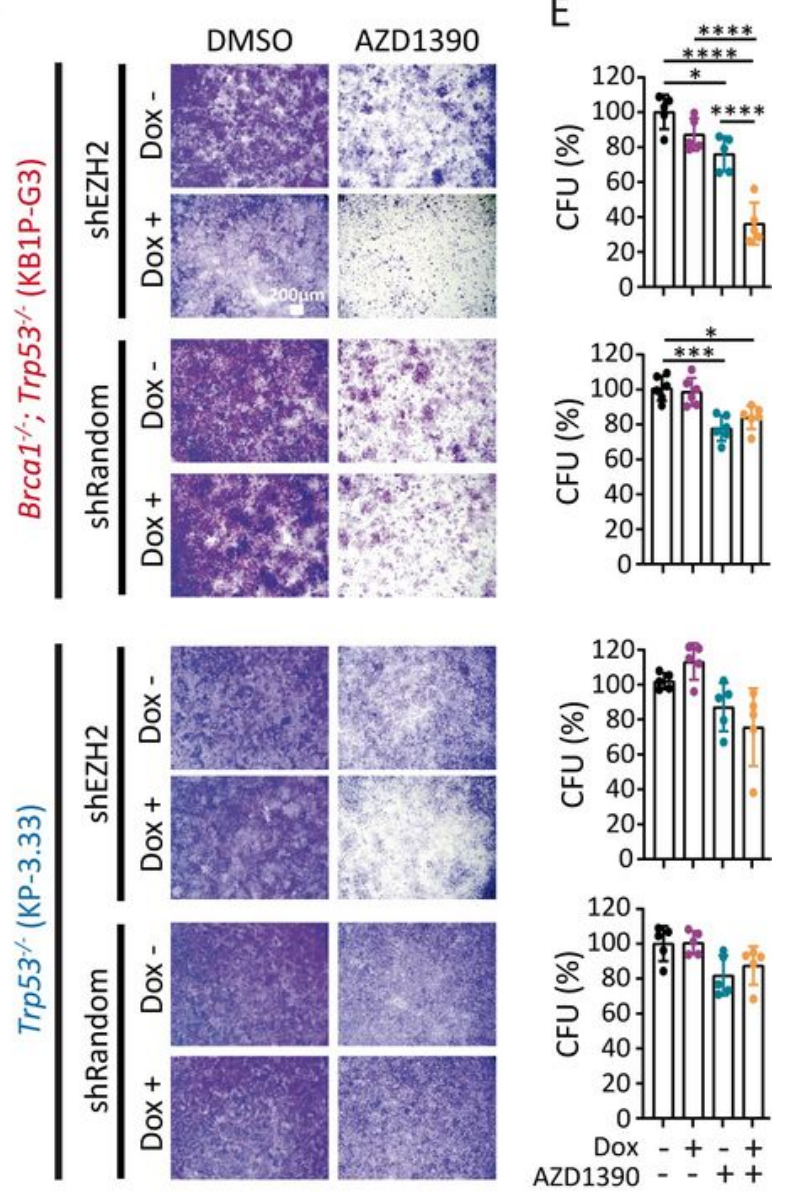

AZD1390 - - + +

\begin{tabular}{|c|c|}
\hline Synergy score & EZH2i/ATMi \\
\hline Brca1 $\% ; \operatorname{Trp} 53^{\%}+$ shEZH2 & $30.5 \pm 13.9 \%$ \\
\hline $\operatorname{Trp} 53^{\%}+$ shEZH2 & $9.4 \pm 11.9 \%$ \\
\hline
\end{tabular}

\begin{tabular}{|c|c|}
\hline Synergy score & EZH2i/ATMi \\
\hline KB1P-G3 & $60.2 \pm 12.1 \%$ \\
\hline KB1P-B11 & $48.5 \pm 16.4 \%$ \\
\hline KP-3.33 & $13.5 \pm 13.8 \%$ \\
\hline KP-6.3 & $10.4 \pm 12.8 \%$ \\
\hline
\end{tabular}

Figure 4

Validation of synergistic EZH2/ATM inhibition using chemically distinct inhibitors and genetic knockdown. (A-B) The synergistic cytotoxic effect is also evident for chemically distinct inhibitors of EZH2 and ATM. The BRCA1-deficient (KB1P-G3 and KB1P-B11) and BRCA1-proficient (KP-3.33 and KP- 
6.3) cell lines were treated with (A) an alternative inhibitor against EZH2 (ZLD1039 at $3 \mu \mathrm{M})$ alone or in combination with AZD1390 $(5 \mu \mathrm{M})$, or (B) an alternative inhibitor against ATM (KU60019 at $5 \mu \mathrm{M})$ alone or in combination with GSK126 $(7.5 \mu \mathrm{M})$, respectively. After 72 hours, cell viability was quantified by CellT iter-Glo assay. Data are presented as mean + SD of at least four independent experiments. Bliss synergy score for ZLD1039/AZD1390: KB1P-G3: $32.2 \pm$ 13.1\%; KB1P-B11: $36.5 \pm$ 10.0\%; KP-3.33: $12.3 \pm$ 9.7\%; KP-6.3: $10.1 \pm$ 9.7\%. Bliss synergy score for GSK126/KU60019: KB1P-G3: $60.2 \pm 12.1 \%$; KB1P-B11: $48.5 \pm 16.4 \%$; KP-3.33: $13.5 \pm 13.8 \%$; KP-6.3: $10.4 \pm 12.8 \%$. (C-E) Genetic ablation of EZH2 mimics pharmacological H3K27me3 inhibition. (C) Western blotting showing Ezh2 knockdown and reduced H3K27me3 levels upon inducible EZH2 knockdown after 7 days of Dox treatment (100 ng/ $\mu$ ). (D) Representative microscopic images of colony formation assay using Dox-induced EZH2 knockdown in KB1P-G3 and KP-3.33-shEzh2 cells are shown at 0.56x magnification. Scale bar $200 \mu \mathrm{m}$. (E) Quantification of crystal violet positive stained colonies is presented as mean \pm SD of five independent experiments. Bliss synergy score: KB1P-G3-shEzh2: $30.5 \pm 13.9 \%$; KP-3.33-shEzh2: $9.4 \pm 11.9 \%$.

Significance was tested by one-way ANOVA with Tukey multiple comparison test. CFU - colony forming unit.

A

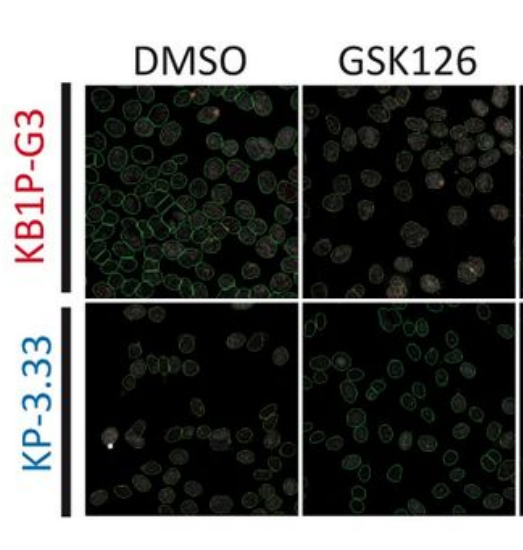

C

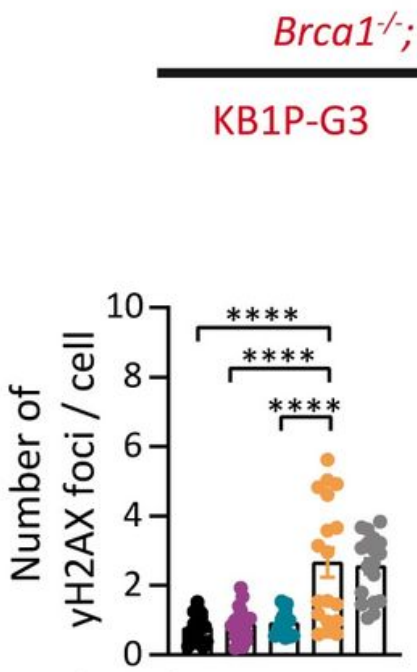

GSK126 (EZH2i) - + - + AZD1390 (ATMi) - - + + Cisplatin - - - +
GSK126 +
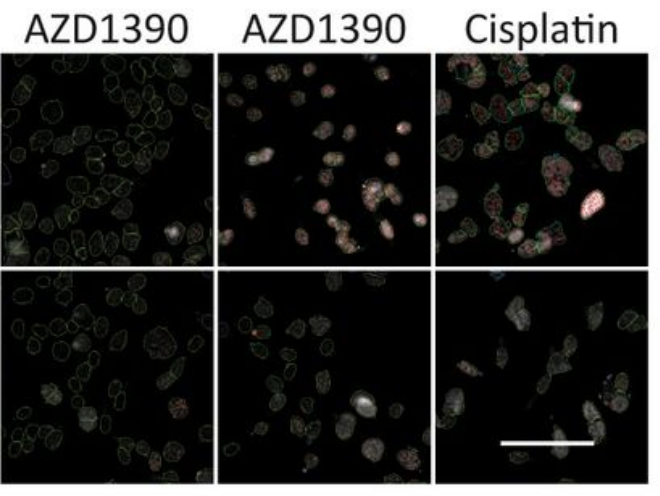

B

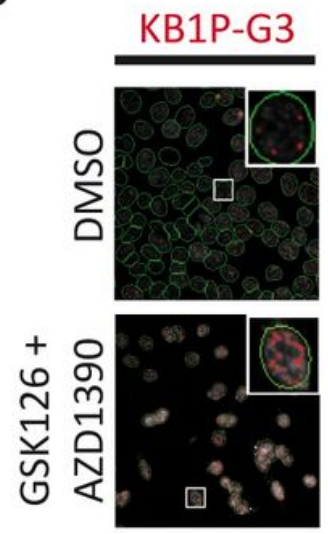

Figure 5 
Combined inhibition of EZH2/ATM induces genotoxic stress. (A) Representative microscopic images of immunofluorescence staining of $\mathrm{YH} 2 \mathrm{AX}$ in the nucleus of cells exposed to GSK126 and AZD1390 for 48 hours. Green or blue encircled cells indicate inclusion or exclusion of objects. Red indicates detected yH2AX foci. Scale bar $100 \mu \mathrm{m}$. (B) Magnified inset of a representative KB1P-G3 cell with (top) high number of yH2AX foci per cell after combined EZH2/ATM inhibition and (bottom) low number of yH2AX foci per cell after DMSO treatment. (C) Quantification of immunofluorescence staining. Values are mean + SEM of at least four independent experiments with each three technical replicates. Bliss synergy score: KB1P-G3: $23.7 \pm$ 10.0\%; KB1P-B11: $21.8 \pm 11.5 \%$; KP-3.33: $2.3 \pm 6.5 \%$; KP-6.3: $1.7 \pm 8.1 \%$. See Supplementary Figure $\mathrm{S} 4$ for bar graphs showing cells per field (upper panels) and numbers of analyzed cells per condition (lower panels). Significance was tested by one-way ANOVA with Tukey multiple comparison test. 
A

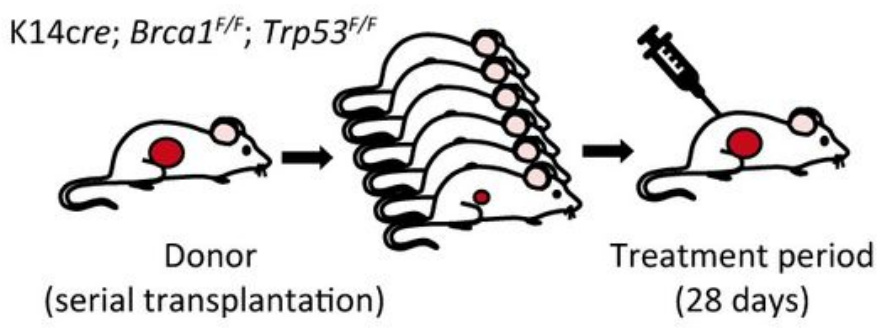

B

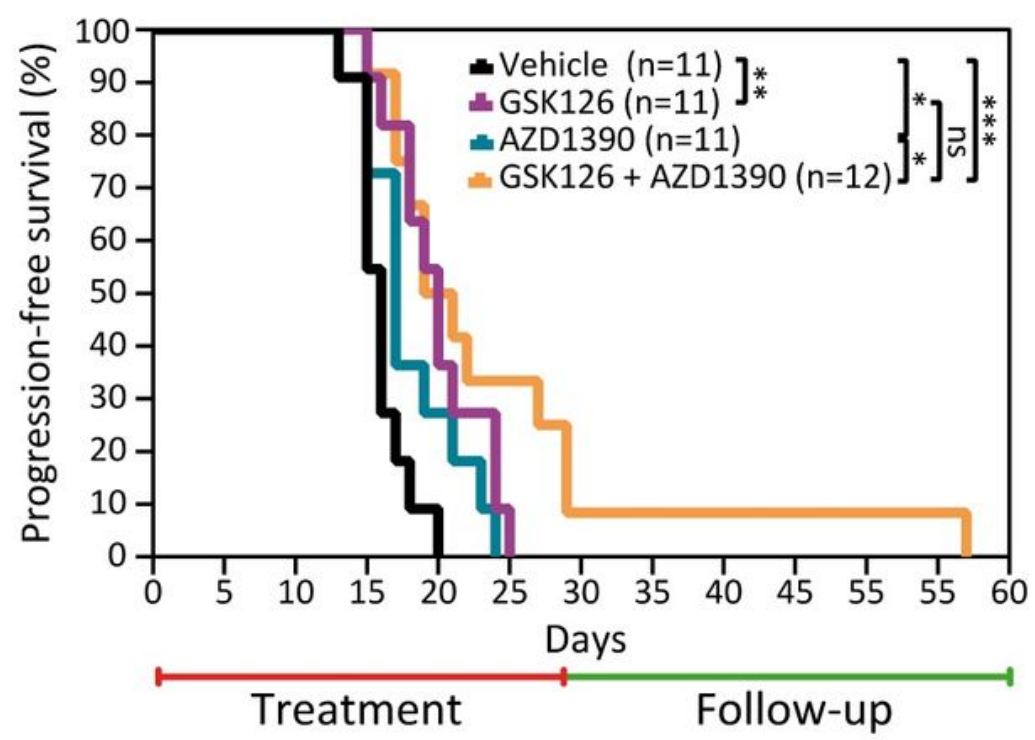

C

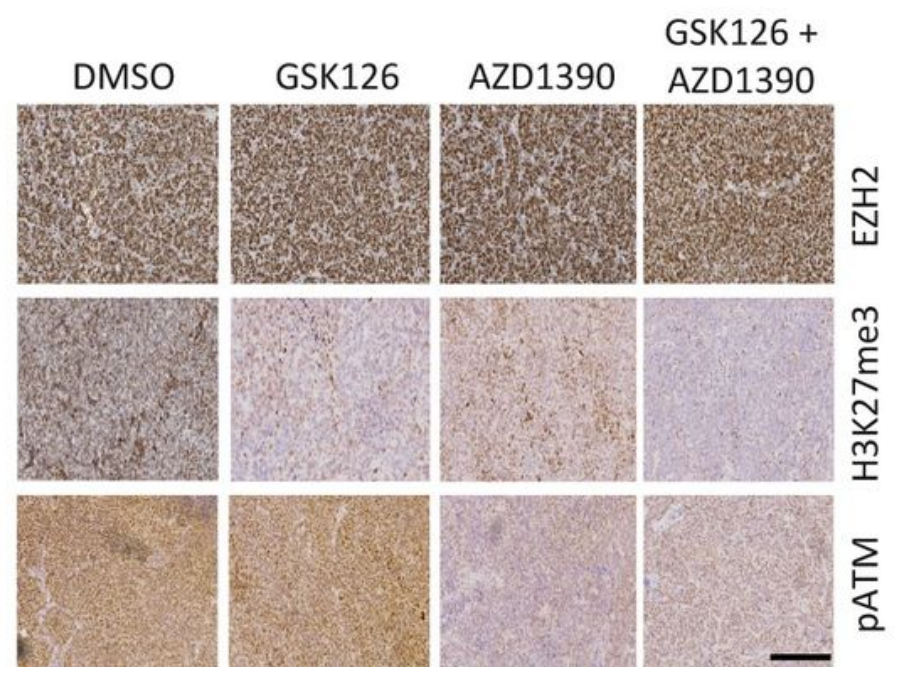

\section{Figure 6}

Combined EZH2/ATM inhibition shows an enhanced anti-tumor effect in KB1P mammary tumor allografts. (A) Mammary tumor tissue fragments from KB1P mice were transplanted into the 4th mammary fat pad of FVB females and treatments were initiated following tumor outgrowth to approximately $100 \mathrm{~mm} 3(100 \%)$. Upon tumor detection (day 0), mice were treated for 28 consecutive days. (B) Progression-free survival curves of KB1P mammary tumor-bearing mice treated with vehicle 
(black), GSK126 (150 mg/kg i.p.) single agent (purple), AZD1390 (2 × $20 \mathrm{mg} / \mathrm{kg}$, oral gavage) single agent (green), or combined treatment with GSK126/AZD1390 (orange). Progression-free survival was defined as the time to develop a 10-time tumor volume increase. Survival curves were generated with the Kaplan-Meier approach and compared with the log-rank test as indicated. (C) Representative images of immunofluorescence staining of EZH2, H3K27me3 and phosphorylated ATM in mammary tumor tissue after treatment as indicated. Scale bar $200 \mu \mathrm{m}$.

\section{BRCA1 proficiency}

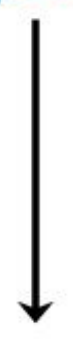

DNA repair by $H R$
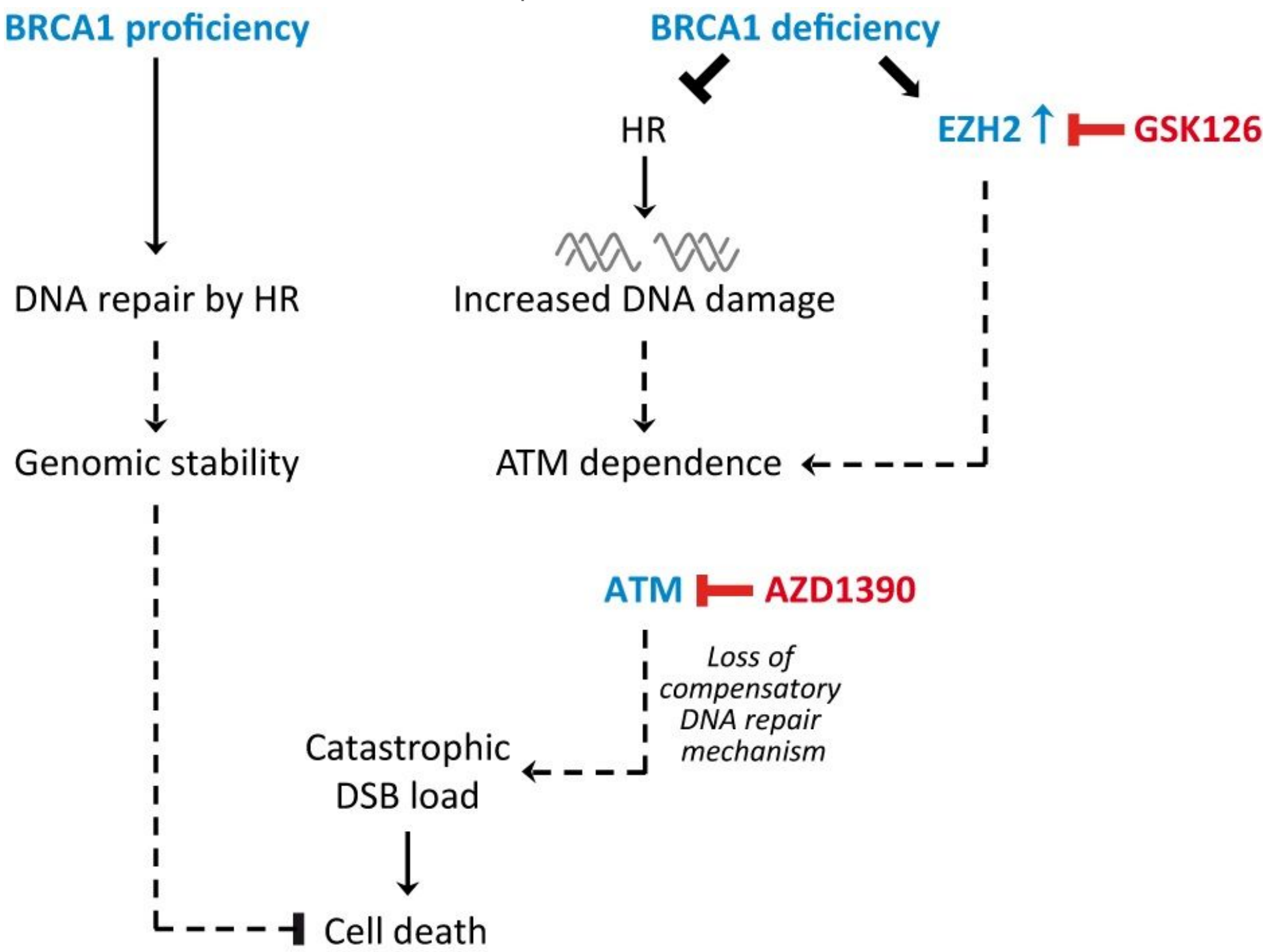

Figure 7

Proposed model for the synthetic lethal mechanism by combined EZH2/ATM inhibition for the treatment of BRCA1-deficient breast cancer. In BRCA1-deficient breast cancer cells, overexpression of EZH2 confers increased survival signaling. GSK126-mediated EZH2 inhibition could lead to ATM dependence. BRCA1deficiency leads to compromised HR and accumulation of DNA DSBs aggravating the ATM dependence. Simultaneous AZD1390-mediated ATM inhibition abolishes cell cycle checkpoint and alternative DNA repair signaling leading to increased $\mathrm{yH} 2 \mathrm{AX}$ foci and apoptosis-mediated cell death. Arrows and stop bars define activation and inhibition, respectively. 


\section{Supplementary Files}

This is a list of supplementary files associated with this preprint. Click to download.

- Additionalfile1SupplFigure1.tif

- Additionalfile2SupplFigure2.tif

- Additionalfile3SupplFigure3.tif

- Additionalfile4SupplFigure4.tif

- Additionalfile5SupplementaryTableS1.xlsx

- Additionalfile6SupplementaryTableS2.xIsx 